\title{
Intramolecular "Hydroiminiumation and -amidiniumation" of Alkenes: A Convenient, Flexible, and Scalable Route to Cyclic Iminium and Imidazolinium Salts
}

\author{
Rodolphe Jazzar, Jean-Baptiste Bourg, Rian D. Dewhurst, Bruno Donnadieu, and Guy \\ Bertrand ${ }^{*}$ \\ UCR-CNRS Joint Research Chemistry Laboratory (UMI2957), Department of Chemistry, University \\ of California, Riverside, California 92521-0403
}

\begin{abstract}
Addition of a stoichiometric amount of $\mathrm{HCl}$ to alkenylaldimines, -formamidines, and -amidines results in the protonation of the $\mathrm{sp}^{2}$-nitrogen atom. The resulting alkenylaldiminium, formamidinium, and -amidinium salts can be isolated and fully characterized, including single-crystal $\mathrm{X}$-ray diffraction studies. Heating solutions of these salts induces ring closure cleanly and regioselectively via formal "exo" addition of the nitrogen-hydrogen bond to the pendent carboncarbon double bond, affording the corresponding cyclic aldiminium, dihydroisoquinolinium, and imidazolinium salts. Of special interest, novel 4,4-disubstituted imidazolinium salts are accessible via this synthetic route. Similarly, addition of phosgene to alkenyl ureas and alkenyl amides, followed by gentle heating, cleanly affords $C$-chloro imidazolinium, and cyclic $C$-chloro iminium salts, respectively. Treatment of the latter with tetrakis(triphenylphosphine)-palladium allows for the preparation of the first transition-metal complex bearing a cyclic arylaminocarbene as ligand. Deuterium labeling experiments suggest that the mechanism of the hydroiminiumation and amidiniumation reactions involves an intramolecular proton transfer to the double bond in the ratedetermining step. This novel synthetic methodology gives access to a variety of $\mathrm{N}$-heterocyclic carbene (NHC) and cyclic alkyl- and arylaminocarbene (CAAC) precursors.
\end{abstract}

\section{Introduction}

Imidazolinium A (Scheme 1) and imidazolium salts have found numerous applications as room-temperature ionic liquids, of particular importance for "Green Chemistry", 1 but also for dye-sensitized solar cells, electrochemical devices, wet double-layer capacitors, and ion transport systems. ${ }^{2}$ When $\mathrm{R}=\mathrm{H}$, they serve as direct precursors for free Arduengo-type carbenes (NHCs) ${ }^{3,4}$ and for the introduction of NHC ligands into transition metal based catalysts; ${ }^{5}$ the chloro analogues $(\mathrm{R}=\mathrm{Cl})$ can also be used for the latter application. ${ }^{6}$ As recently noted by Fürstner et al., 7 despite a huge number of structural variants, several obvious and seemingly trivial substitution patterns for these dinitrogen-containing heterocyclic salts are as yet unknown or very rare. Among them are the unsymmetrical versions, and particularly 4,4disubstituted imidazolinium salts $\mathbf{A}^{\prime}$. 7,8 These limitations stem from the scarcity of viable synthetic routes. Moreover, it is astounding to note that the classical route to imidazolinium salts 9 involves the use of $\mathrm{NaBH}_{4}$ as a reducing agent, which turns out to be rather expensive from an industrial point of view, and even more importantly from a "Green Chemistry"

gbertran@ucr.Edu.

Supporting Information Available: X-ray crystallographic data for 4b, 10a, 11a, and 18a (CIF) and spectral data for prepared compounds. This material is available free of charge via the Internet at http://pubs.acs.org. 
perspective, poses many environmental and recycling challenges. Among the alternative synthetic methods, ${ }^{10}$ the most common relies on $\mathrm{N}$-substitution of appropriate imidazoline precursors, which is only possible with a small number of reactive electrophiles.

Because saturated nitrogen-containing heterocycles form the core structures, and are key intermediates, of many natural products, ${ }^{11}$ several synthetic methods for their preparation have been developed. Among them is the intramolecular hydroamination of alkenes, in which the nitrogen-carbon bond is formed by addition of an amine N-H bond to an olefin. ${ }^{12}$ Various catalysts have been used to effect this transformation, and importantly, it has been shown recently that when weakly basic amines are used, the hydroamination can be catalyzed by Brönsted acids. ${ }^{13}$ Recognizing that imines are less basic than amines, we have studied the feasibility of "hydroiminiumation" reactions and recently reported preliminary results. ${ }^{14} \mathrm{We}$ showed that the addition of a stoichiometric amount of $\mathrm{HCl}$ to alkenyl imines resulted in the formation of the $\mathrm{N}$-protonated species $\mathbf{B}$, which underwent, under gentle heating, a ring closure cleanly and regioselectively, leading to the cyclic iminium salts $\mathbf{C}$ (Scheme 1). Importantly, salts $\mathbf{C}$ are the direct precursors of stable cyclic alkylaminocarbenes (CAACs). ${ }^{15}$ We have shown that CAACs can compete with NHCs as ligands for transition-metal-based catalysts $15 \mathrm{a}$ and also allow the preparation of very low coordinate transition-metal centers. ${ }^{15 b}$

We report herein examples of an asymmetric version of the hydroiminiumation reaction and the extension of our approach to the synthesis of dihydroisoquinolinium salts as well as cyclic $C$-chloro iminium salts. We show that the $\mathrm{N}-\mathrm{H}$ bond of amidinium salts also adds to a pendent carbon-carbon double bond giving a straightforward, atom-economical route to imidazolinium salts, especially those of type $\mathbf{A}^{\prime}$. Last, the mechanism of this ring-closing reaction is discussed on the basis of isotopic labeling experiments.

\section{Results and Discussion}

We have already shown that relatively bulky electrophiles (such as the 1,2-epoxy-2methylpropane) approach the cyclohexane moiety of the aza-allyl anion prepared from the aldimine la [derived from (-)-menthone] exclusively from the equatorial direction. ${ }^{15 \mathrm{a}}$ Therefore, despite the fact that la exists as a 90/10 mixture of diastereomers, addition of 3bromo-2-methylpropene to its lithium salt led to the enantiomerically pure alkenylaldimine 2a in 94\% yield (Scheme 2). After addition of $\mathrm{HCl}$ to form the alkenylaldiminium salt 3a, the cyclization occurred readily and was complete after $5 \mathrm{~h}$ at $50{ }^{\circ} \mathrm{C}$. The optically pure cyclic iminium salt $\mathbf{4 a}$ was isolated in $92 \%$ yield (by the previously reported method, $\mathbf{4 a}$ was obtained in only $41 \%$ yield).

The use of 3-bromopropene provided an opportunity to create a new stereogenic center next to nitrogen and to check if the presence of the enantiomerically pure menthyl ring would produce an asymmetric induction. It was pleasing to observe that heating $\mathbf{3 b}$ in acetonitrile at $100{ }^{\circ} \mathrm{C}$ for $12 \mathrm{~h}$ cleanly afforded the desired heterocyclic salt $\mathbf{4 b}$, isolated in $86 \%$ yield, with an $82 \%$ de (according to ${ }^{1} \mathrm{H}$ NMR spectroscopy) (Scheme 2). The absolute configuration of the major diastereomer was assigned by single-crystal X-ray crystallography (Figure 1).

To demonstrate further the generality of the hydroiminiumation route, we turned our attention to the preparation of 3,4-dihydroisoquinolinium salts. Although several synthetic routes are available for such heterocycles, 6,6-disubstituted derivatives featuring a bulky substituent at nitrogen are attainable only through addition of an electrophile to the nitrogen of the corresponding dihydroisoquinoline, thus placing severe restrictions on the size of the $\mathrm{N}$ substituent; ${ }^{16}$ they are highly desirable precursors for the preparation of the as yet unknown stable cyclic arylaminocarbenes. ${ }^{17}$ Starting from the readily available aldimine $\mathbf{5}$, a lithiumhalogen exchange with $n$-BuLi, followed by addition of 3-bromo-2-methylpropene 
quantitatively led to the desired precursor 6 . Then, addition of $\mathrm{HCl}$ and heating at $110{ }^{\circ} \mathrm{C}$ for $24 \mathrm{~h}$ afforded the 6,6-dimethyldihydroisoquinolinium salt 7 , which was isolated in $95 \%$ yield (Scheme 3).

Despite the greater basicity of amidines over imines, it was decided to investigate the possibility of extrapolating this ring-closure protocol to the synthesis of imidazolinium salts $\mathbf{A}$. Deprotonation of 8a with $n$-BuLi followed by addition of allyl chloride afforded the corresponding alkenyl formamidine 9a in 94\% yield (Scheme 4). Addition of a stoichiometric amount of a $2 \mathrm{M}$ solution of $\mathrm{HCl} / \mathrm{Et}_{2} \mathrm{O}$ to a toluene or ether solution of $9 \mathrm{a}$ resulted in the formation of a precipitate. After filtration and recrystallization from chloroform, a new compound 10a was isolated as white crystals in $95 \%$ yield. A doublet resonance in the ${ }^{1} \mathrm{H}$ NMR spectrum at $14.0 \mathrm{ppm}\left(J_{\mathrm{HH}}=12.0 \mathrm{~Hz}\right)$ suggested the protonation of one nitrogen atom. A single-crystal X-ray diffraction study unambiguously proved the alkenyl formamidinium structure of 10a (Figure 2, left). We were pleased to observe that heating a toluene or acetonitrile solution of 10a in a flask sealed by a Teflon stopcock at $135{ }^{\circ} \mathrm{C}$ for $36 \mathrm{~h}$ afforded the desired imidazolinium salt 11a as a racemate in $83 \%$ yield (Figure 2, right) (Scheme 4). Similarly, alkenylformamidines $\mathbf{9 b - d}$, prepared from the corresponding formamidines in high yields, were found to be suitable precursors for 4,4-disubstituted imidazolinium salts 11b-d. The protonation/cyclization sequence was performed in situ. Imidazolinium salt 11b was isolated in $80 \%$ yield after heating for $24 \mathrm{~h}$ at $110^{\circ} \mathrm{C}$. When the Dipp substituents of $10 \mathrm{~b}$ were replaced by mesityl groups, the cyclization required heating at $135^{\circ} \mathrm{C}$ for $12 \mathrm{~h}$, and heterocyclic salt 11c was isolated in 78\% yield. Under the same experimental conditions, imidazolinium salt 11d featuring 2,6-difluorophenyl groups was also obtained in 79\% yield from 9d, illustrating the broad scope of application of this synthetic route.

Since the $N$-substitution of formamidines by reactive electrophiles is a well-known reaction, it quickly became apparent that the above synthesis of imidazolinium salts could be simplified even further. Indeed, allyl bromide and 3-bromo-2-methylpropene are quite reactive as electrophiles, and in the process of the substitution of formamidine 8a, one equivalent of $\mathrm{HBr}$ is liberated, protonating the remaining nitrogen. Thus, heating an equimolar toluene solution of allyl bromide and formamidine 8a in a tube sealed by a Teflon stopcock at $135^{\circ} \mathrm{C}$ for $36 \mathrm{~h}$ afforded imidazolinium salt 11a, which was isolated in $66 \%$ yield. The one-pot procedure appeared to occur at a slightly lower temperature when 3-bromo-2-methylpropene was used. The reaction was complete after $24 \mathrm{~h}$ at $110^{\circ} \mathrm{C}$ and imidazolinium salt 11b, featuring two methyl groups in the 4 position, was isolated in $70 \%$ yield (Scheme 5).

The cyclization process is not restricted to formamidines. When a phenyl substituent is present at the NCN carbon and given bulky groups at nitrogen, the allylation of $\mathbf{1 2}$ may be performed via its corresponding lithium salt. Then, after protonation with $\mathrm{HCl}$, the ring closure leading to $\mathbf{1 3}$ is complete after $12 \mathrm{~h}$ at $105^{\circ} \mathrm{C}$ (Scheme 6).

As mentioned in the introduction, $C$-chloro imidazolinium salts can be used to introduce the corresponding NHCs as ligands for transition-metal centers. Chloro amidines are classically prepared by addition of a strong chlorinating reagent (such as phosgene or oxalyl chloride) to trisubstituted urea derivatives, followed by elimination of $\mathrm{HCl}$; obviously, this transformation proceeds via $C$-chloro amidinium salts. ${ }^{18}$ Therefore, we hypothesized that using $N$-allylurea 14 as a starting material, a $C$-chloro amidinium salt would be formed by addition of phosgene and this may undergo a subsequent hydroamidiniumation reaction, affording the desired $C$ chloro imidazolinium salt 15. $N, N^{\prime}$-Diaryl- $N$-allylurea $\mathbf{1 4}$ is readily available as shown in Scheme 7. Simple heating of a toluene solution of 14 at $80{ }^{\circ} \mathrm{C}$ for $24 \mathrm{~h}$ in the presence of a stoichiometric amount of phosgene, cleanly leads to $\mathbf{1 5}$, which was isolated in $78 \%$ yield. 
A very similar methodology can be applied to prepare $C$-chloro iminium salts 17a,b. Heating 16a and $16 \mathbf{b}$ at $80{ }^{\circ} \mathrm{C}$ for $24 \mathrm{~h}$ gave rise to $\mathbf{1 7 a}$ and $\mathbf{1 7 b}$, which were isolated in 86 and $78 \%$ yield, respectively (Scheme 8 ). Since transition-metal complexes of cyclic aminoarylcarbenes have not yet been reported, and as a proof of principle that this type of $C$-chloro iminum salts are effective proligands for transition-metal complexes, $\mathbf{1 7 a}$ was treated with palladium tetrakis (triphenylphosphine). Indeed, palladium(II) complex 18a was isolated in 55\% yield and fully characterized including a single-crystal X-ray diffraction study (Figure 3).

In order to gain more insight into the mechanism of the cyclization process, deuterium labeling experiments were carried out using $\mathbf{2 c}$ and $\mathbf{9 a}$ as starting materials. In both cases, only one deuterated compound $\left(\mathbf{4}_{\mathbf{D}}\right.$ and $\mathbf{1 1} \mathbf{c}_{\mathbf{D}}$, respectively) was obtained (Scheme 9 ). These observations argue against a reversible protonation of the alkene, since in this case mono-and polydeuterated derivatives would be obtained. Of special importance is the exclusive formation of $\mathbf{4 c}_{\mathbf{D}}$. If an inter-molecular protonation of the olefin were to occur, we would expect little or no preference for deuteration at either olefinic carbon, and consequently observe scrambling of the deuterium over these two positions, since in both cases a secondary carbocation would be formed. Therefore, it can be concluded that the proton is transferred intramolecularly to the double bond in the rate-determining step.

\section{Conclusion}

Simple protonation of the $\mathrm{sp}^{2}$-nitrogen atom of alkenylaldimines, -formamidines, and amidines and addition of a chlorinating agent to alkenyl amides followed by heating are very convenient synthetic routes to a variety of NHC and CAAC precursors. Deuterium labeling experiments suggest that the mechanism of the cyclization reaction involves an intramolecular proton transfer to the double bond in the rate-determining step. Extension of this novel methodology to related systems is under investigation.

\section{Experimental Section}

\section{General Methods}

All manipulations were performed under an inert atmosphere of dry argon using standard Schlenk techniques. Dry, oxygen-free solvents were employed. ${ }^{1} \mathrm{H}$ and ${ }^{13} \mathrm{C}$ NMR chemical shifts are reported relative to residual solvent resonances. ${ }^{31} \mathrm{P}$ and ${ }^{19} \mathrm{~F}$ NMR chemical shifts are reported relative to $85 \% \mathrm{H}_{3} \mathrm{PO}_{4}$ and $\mathrm{CFCl}_{3}$, respectively.

\section{Synthesis of Aldimine 1a}

In a Schlenk tube containing activated molecular sieves (10 g), menthyl 3-carboxaldehyde $(90 / 10 \text { mixture of diastereomers })^{19}(33.8 \mathrm{~mL}, 181 \mathrm{mmol})$ was added dropwise at room temperature to a solution of 2,6-diisopropylaniline $(30.6 \mathrm{~g}, 173 \mathrm{mmol})$ in toluene $(100 \mathrm{~mL})$. The suspension was stirred for $12 \mathrm{~h}$ at $100^{\circ} \mathrm{C}$. After filtration, the molecular sieves were washed with hexane $(60 \mathrm{~mL})$. Evaporation of the solvent, and heating under vacuum at $100{ }^{\circ} \mathrm{C}$ to remove all volatiles, afforded 1a $(80 \%$ de) as an oily yellow solid $(52.0 \mathrm{~g}, 92 \%)$. Major diastereomer: ${ }^{1} \mathrm{H}$ NMR $\left(\mathrm{CDCl}_{3}\right) \delta 7.43\left(\mathrm{~d}, J_{\mathrm{HH}}=7.1 \mathrm{~Hz}, 1 \mathrm{H}\right), 7.14-7.05(\mathrm{~m}, 3 \mathrm{H}), 2.98$ (sept, $\left.J_{\mathrm{HH}}=6.9 \mathrm{~Hz}, 2 \mathrm{H}\right), 2.54(\mathrm{~m}, 1 \mathrm{H}), 1.94-1.70(\mathrm{~m}, 4 \mathrm{H}), 1.59-1.38(\mathrm{~m}, 5 \mathrm{H}), 1.17$ ( $2 \times$ overlapping $\left.\mathrm{d}, J_{\mathrm{HH}}=6.9 \mathrm{~Hz}, 12 \mathrm{H}\right), 0.97\left(2 \times\right.$ overlapping d, $\left.J_{\mathrm{HH}}=6.5 \mathrm{~Hz}, 6 \mathrm{H}\right), 0.90\left(\mathrm{~d}, J_{\mathrm{HH}}=6.9 \mathrm{~Hz}\right.$, $\left.{ }_{3}^{3} \mathrm{H}\right) ;{ }^{13} \mathrm{C}\left\{{ }^{1} \mathrm{H}\right\}$ NMR $\left(\mathrm{CDCl}_{3}\right) \delta 171.6,148.9,138.0,124.1,123.1,48.5,45.2,39.2,35.0,32.3$, 29.5, 27.7, 23.9, 23.7, 22.8, 21.6,15.5; MS(HR-ESI) $\mathrm{m} / 2328.3004[\mathrm{M}+\mathrm{H}]+($ calcd 328.3004).

\section{Synthesis of Alkenylaldimine 2a}

An $\mathrm{Et}_{2} \mathrm{O}$ solution $(20 \mathrm{~mL})$ of $\mathrm{Me}_{2} \mathrm{NLi}(0.467 \mathrm{~g}, 9.2 \mathrm{mmol})$ at $-78^{\circ} \mathrm{C}$ was added to an $\mathrm{Et}_{2} \mathrm{O}$ solution $(20 \mathrm{~mL})$ of $1 \mathbf{a}(3.00 \mathrm{~g}, 9.2 \mathrm{mmol})$ at $-78{ }^{\circ} \mathrm{C}$. After $15 \mathrm{~min}$, the mixture was warmed 
to $\mathrm{rt}$ and stirred for an additional $4 \mathrm{~h}$. The volatiles were removed under vacuum, affording an oily yellow/orange residue which was dissolved in $\mathrm{Et}_{2} \mathrm{O}(30 \mathrm{~mL})$ and cooled to $-78{ }^{\circ} \mathrm{C}$. 3-

Bromo-2-methylpropene $(0.92 \mathrm{~mL}, 9.2 \mathrm{mmol})$ was slowly added. After $15 \mathrm{~min}$, the solution was warmed to rt and stirred for an additional $12 \mathrm{~h}$. Removal of the volatiles under vacuum and extraction with hexanes afforded alkenylaldimine $\mathbf{2 a}$ as a light yellow oil $(3.11 \mathrm{~g}$, 94\%): ${ }^{1} \mathrm{H} \mathrm{NMR}\left(\mathrm{CDCl}_{3}\right) \delta 8.21(\mathrm{~s}, 1 \mathrm{H}), 7.33\left(\mathrm{~d}, J_{\mathrm{HH}}=7.2 \mathrm{~Hz}, 2 \mathrm{H}\right), 7.27\left(\mathrm{t}, J_{\mathrm{HH}}=7.2 \mathrm{~Hz}, 1 \mathrm{H}\right)$, 5.23 and $5.04(\mathrm{~s} \times 2,1 \mathrm{H}), 3.22\left(\mathrm{sept}, J_{\mathrm{HH}}=6.8 \mathrm{~Hz}, 2 \mathrm{H}\right), 2.84(\mathrm{~m}, 2 \mathrm{H}), 2.53\left(\mathrm{~m}, J_{\mathrm{HH}}=6.4 \mathrm{~Hz}\right.$, $1 \mathrm{H}), 2.23\left(\mathrm{~d}, J_{\mathrm{HH}}=12.8 \mathrm{~Hz}, 1 \mathrm{H}\right), 2.12(\mathrm{~s}, 3 \mathrm{H}), 2.07\left(\mathrm{~d}, J_{\mathrm{HH}}=12.4 \mathrm{~Hz}, 1 \mathrm{H}\right), 1.89\left(\mathrm{~d}, J_{\mathrm{HH}}=\right.$ $12.4 \mathrm{~Hz}, 1 \mathrm{H}), 1.77-1.55(\mathrm{~m}, 4 \mathrm{H}), 1.42(\mathrm{~m}, 12 \mathrm{H}), 1.22\left(\mathrm{~d}, J_{\mathrm{HH}}=6.8 \mathrm{~Hz}, 3 \mathrm{H}\right), 1.16\left(\mathrm{~d}, J_{\mathrm{HH}}=\right.$ $6.4 \mathrm{~Hz}, 3 \mathrm{H}), 1.02\left(\mathrm{~d}, J_{\mathrm{HH}}=6.8 \mathrm{~Hz}, 3 \mathrm{H}\right) ;{ }^{13} \mathrm{C}\left\{{ }^{1} \mathrm{H}\right\} \mathrm{NMR}\left(\mathrm{CDCl}_{3}\right) \delta 172.1,149.3,142.8,137.7$, 123.9, 123.1, 116.4, 49.2, 47.7, 45.7, 44.1, 35.8, 29.3, 27.8, 26.5, 26.0, 24.0, 23.9, 23.7, 23.2, 23.1, 19.1; MS(EI) $m / 2382[\mathrm{M}+\mathrm{H}]+$.

\section{Synthesis of Alkenylaldimine $\mathbf{2 b}$}

Following the same procedure used for $\mathbf{2 a}$, but with 3-bromopropene, $\mathbf{2 b}$ was obtained as a light yellow oil (89\%): ${ }^{1} \mathrm{H}$ NMR $\left(\mathrm{CDCl}_{3}\right) \delta 7.98(\mathrm{~s}, 1 \mathrm{H}), 7.16-7.06(\mathrm{~m}, 3 \mathrm{H}), 6.02-5.88(\mathrm{~m}$, $1 \mathrm{H}), 5.20(\mathrm{~s}, 1 \mathrm{H}), 5.16(\mathrm{~s}, 1 \mathrm{H}), 2.97$ (sept, $\left.J_{\mathrm{HH}}=6.8 \mathrm{~Hz}, 2 \mathrm{H}\right), 2.67-2.45(\mathrm{~m}, 2 \mathrm{H}), 2.26-2.15$ $(\mathrm{m}, 1 \mathrm{H}), 2.00-1.66(\mathrm{~m}, 4 \mathrm{H}), 1.46-1.26(\mathrm{~m}, 4 \mathrm{H}), 1.20\left(\mathrm{t}, J_{\mathrm{HH}}=6.4 \mathrm{~Hz}, 12 \mathrm{H}\right), 1.00\left(\mathrm{~d}, J_{\mathrm{HH}}=\right.$ $6.9 \mathrm{~Hz}, 3 \mathrm{H}), 1.16\left(\mathrm{~d}, J_{\mathrm{HH}}=6.2 \mathrm{~Hz}, 3 \mathrm{H},\right), 1.02\left(\mathrm{~d}, J_{\mathrm{HH}}=6.9 \mathrm{~Hz}, 3 \mathrm{H}\right) ;{ }^{13} \mathrm{C}\left\{{ }^{1} \mathrm{H}\right\} \mathrm{NMR}$ $\left(\mathrm{CDCl}_{3}\right) \delta 171.9,149.3,137.6,134.7,123.9,123.1,118.4,48.4,48.1,44.9,41.8,35.8,29.2$, 27.7, 25.6, 24.4, 23.8, 23.7, 23.3, 23.0,19.2; MS(EI) $\mathrm{m} / z 368$ [M + H]+.

\section{Synthesis of Alkenylaldiminium 3a}

To a hexane solution $(10 \mathrm{~mL})$ of $2 \mathrm{a}(1.00 \mathrm{~g}, 2.6 \mathrm{mmol})$ at $-78{ }^{\circ} \mathrm{C}$ was added a solution of $\mathrm{HCl}$ in $\mathrm{Et}_{2} \mathrm{O}(1.30 \mathrm{~mL}, 2.0 \mathrm{M}, 2.6 \mathrm{mmol})$. Precipitation of a white powder was immediately observed. After $15 \mathrm{~min}$, the mixture was warmed to rt and stirred for an additional $15 \mathrm{~min}$. Filtration of the precipitate, washing with hexanes $(2 \times 10 \mathrm{~mL})$, and drying under vacuum afforded the alkenyliminium salt $3 \mathbf{a}$ as a white powder $(1.01 \mathrm{~g}, 92 \%)$ : nip $60-62{ }^{\circ} \mathrm{C} \mathrm{dec} ;{ }^{1} \mathrm{H}$ NMR $\left(\mathrm{CDCl}_{3}\right) \delta 14.66$ (br s, 1H), $8.31(\mathrm{~s}, 1 \mathrm{H}), 7.24(\mathrm{~m}, 3 \mathrm{H}), 5.08(\mathrm{~s}, 1 \mathrm{H}), 4.93(\mathrm{~s}, 1 \mathrm{H}), 3.07$ $\left(\mathrm{d}, J_{\mathrm{HH}}=13.7 \mathrm{~Hz}, 1 \mathrm{H}\right), 2.95\left(\mathrm{sept}, J_{\mathrm{HH}}=6.8 \mathrm{~Hz}, 2 \mathrm{H}\right), 2.73\left(\mathrm{~d}, J_{\mathrm{HH}}=13.7 \mathrm{~Hz}, 1 \mathrm{H}\right), 2.43(\mathrm{br}$ d, $\left.J_{\mathrm{HH}}=13.0 \mathrm{~Hz}, 1 \mathrm{H}\right), 2.30\left(\mathrm{sept}, J_{\mathrm{HH}}=6.4 \mathrm{~Hz}, 2 \mathrm{H}\right), 1.95(\mathrm{~s}, 2 \mathrm{H}), 1.86-1.50(\mathrm{~m}, 6 \mathrm{H}), 1.34$ $(\mathrm{m}, 2 \mathrm{H}), 1.26\left(\mathrm{dd}, J_{\mathrm{HH}}=2.3 \mathrm{~Hz}, 12 \mathrm{H}\right), 0.99\left(\mathrm{br} \mathrm{t}, J_{\mathrm{HH}}=7.9 \mathrm{~Hz}, 6 \mathrm{H}\right), 0.79\left(\mathrm{~d}, J_{\mathrm{HH}}=6.8 \mathrm{~Hz}\right.$, $3 \mathrm{H}) ;{ }^{13} \mathrm{C}\left\{{ }^{1} \mathrm{H}\right\} \mathrm{NMR}\left(\mathrm{CDCl}_{3}\right) \delta 189.9,143.2,139.2,134.8,130.7,124.7,119.2$,

$51.7,46.6,45.7,42.4,34.8,30.7,28.8,26.1,26.0,24.2,23.9,23.4,23.2,22.5,18.3$.

\section{Synthesis of Alkenylaldiminium Salt 3b}

Following the same procedure used for $\mathbf{3 a}$, but starting from $\mathbf{2 b}(1.00 \mathrm{~g}, 2.7 \mathrm{mmol}), \mathbf{3 b}$ was obtained as a white solid $(0.99 \mathrm{~g}, 90 \%)$ : nip $60{ }^{\circ} \mathrm{C} ;{ }^{1} \mathrm{H}$ NMR $\left(\mathrm{CDCl}_{3}\right) \delta 15.47$ (br s, $\left.1 \mathrm{H}\right), 8.15$ $(\mathrm{s}, 1 \mathrm{H}), 7.26\left(\mathrm{t}, J_{\mathrm{HH}}=7.6 \mathrm{~Hz}, 1 \mathrm{H}\right), 7.13\left(\mathrm{~d}, J_{\mathrm{HH}}=7.6 \mathrm{~Hz}, 2 \mathrm{H}\right), 5.75(\mathrm{~m}, 1 \mathrm{H}), 5.21\left(\mathrm{~d}, J_{\mathrm{HH}}=\right.$ $2.7 \mathrm{~Hz}, 1 \mathrm{H},=\mathrm{CH}_{2}$ ), $5.19(\mathrm{br} \mathrm{d}, 1 \mathrm{H}), 3.08\left(\mathrm{dd}, J_{\mathrm{HH}}=7.1,13.9 \mathrm{~Hz}, 1 \mathrm{H}\right), 2.84\left(\mathrm{sept}, J_{\mathrm{HH}}=6.7\right.$ $\mathrm{Hz}, 2 \mathrm{H}), 2.66\left(\mathrm{dd}, J_{\mathrm{HH}}=7.1,13.9 \mathrm{~Hz}, 1 \mathrm{H}\right), 2.44\left(\mathrm{dd}, J_{\mathrm{HH}}=12.8,13.9 \mathrm{~Hz}, 1 \mathrm{H}\right), 2.08$ (sept, $\left.J_{\mathrm{HH}}=6.5 \mathrm{~Hz}, 1 \mathrm{H}\right), 1.79\left(\mathrm{~d}, J_{\mathrm{HH}}=12.5 \mathrm{~Hz}, 1 \mathrm{H}\right), 1.69\left(\mathrm{~d}, J_{\mathrm{HH}}=12.5 \mathrm{~Hz}, 1 \mathrm{H}\right), 1.53(\mathrm{br} \mathrm{s}, 1 \mathrm{H})$, $1.45\left(\mathrm{~d}, J_{\mathrm{HH}}=13.0 \mathrm{~Hz}, 2 \mathrm{H}\right), 1.22(\mathrm{br} \mathrm{s}, 1 \mathrm{H}), 1.16\left(\mathrm{~d}, J_{\mathrm{HH}}=6.7 \mathrm{~Hz}, 12 \mathrm{H}\right), 0.96(\mathrm{br} \mathrm{s}, 1 \mathrm{H}), 0.99$ $\left(\mathrm{d}, J_{\mathrm{HH}}=6.5 \mathrm{~Hz}, 6 \mathrm{H}\right), 0.69\left(\mathrm{~d}, J_{\mathrm{HH}}=6.7 \mathrm{~Hz}, 3 \mathrm{H}\right) ;{ }^{13} \mathrm{C}\left\{{ }^{1} \mathrm{H}\right\} \mathrm{NMR}\left(\mathrm{CDCl}_{3}\right) \delta 185.1,141.5$, 138.0, 131.3, 128.8, 124.0, 120.6, 50.0, 47.3, 44.7, 40.5, 34.8, 29.9, 28.3, 25.4, 23.7, 23.5, 22.9, 22.3, 18.3.

\section{Synthesis of CAAC, $\mathrm{H}^{+} \mathbf{4 a}$}

An oven-dried, argon-flushed, scalable Schlenk tube with a Teflon stopcock was loaded with 3a (1.00 g, $2.4 \mathrm{mmol})$ in $\mathrm{CHCl}_{3}(5 \mathrm{~mL})$. The mixture was heated at $55^{\circ} \mathrm{C}$ for $6 \mathrm{~h}$. The volatiles 
were removed under vacuum to afford $\mathbf{4 a}$ as a white powder $(0.92 \mathrm{~g}, 92 \%)$ : nip $157{ }^{\circ} \mathrm{C}$; the spectroscopic data are similar to those reported for the trifluoromethane sulfonate salt. ${ }^{15 \mathrm{a}}$

\section{Synthesis of CAAC, $\mathrm{H}^{+} \mathbf{4 b}$}

Cyclic iminiuni salt $\mathbf{4 b}$ was prepared in one pot starting from $\mathbf{2 b}$. An oven-dried, argon-flushed, scalable Schlenk tube with a Teflon stopcock was loaded with $\mathbf{2 b}(1.00 \mathrm{~g}, 2.7 \mathrm{mmol})$ and toluene $(10 \mathrm{~mL})$ and was cooled to $-78{ }^{\circ} \mathrm{C}$, at which point a solution of $\mathrm{HCl}$ in $\mathrm{Et}_{2} \mathrm{O}(1.36 \mathrm{~mL}, 2.0 \mathrm{M}$, $2.7 \mathrm{mmol}$ ) was added. Precipitation of a white powder (3b) was immediately observed. After $15 \mathrm{~min}$ at $-78^{\circ} \mathrm{C}$, the mixture was allowed to warm to rt and stirred for an additional $15 \mathrm{~min}$. The mixture was heated at $110^{\circ} \mathrm{C}$ for $24 \mathrm{~h}$, after which time the volatiles were removed under vacuum to afford $\mathbf{4 b}$ as a $91: 9$ ratio of diastereomers $(0.86 \mathrm{~g}, 86 \%)$. The major isonier was obtained optically pure by recrystallization from chloroform at $-30{ }^{\circ} \mathrm{C}$ : nip $150{ }^{\circ} \mathrm{C} ;{ }^{1} \mathrm{H} \mathrm{NMR}$ $\left(\mathrm{CD}_{3} \mathrm{CN}\right) \delta 10.45(\mathrm{~s}, 1 \mathrm{H}), 7.60\left(\mathrm{t}, J_{\mathrm{HH}}=7.8 \mathrm{~Hz}, 1 \mathrm{H}\right), 7.46-7.42(\mathrm{~m}, 2 \mathrm{H}), 4.87\left(\mathrm{sext}, J_{\mathrm{HH}}=7.1\right.$ $\mathrm{Hz}, 1 \mathrm{H}), 2.97$ (sept, $\left.J_{\mathrm{HH}}=6.8 \mathrm{~Hz}, 2 \mathrm{H}\right), 2.67-2.45(\mathrm{~m}, 2 \mathrm{H}) 2.26-2.15(\mathrm{~m}, 1 \mathrm{H}), 2.00-1.66(\mathrm{~m}$, $4 \mathrm{H}), 1.46-1.26(\mathrm{~m}, 4 \mathrm{H}), 1.20\left(\mathrm{t}, J_{\mathrm{HH}}=6.4 \mathrm{~Hz}, 12 \mathrm{H}\right), 1.00\left(\mathrm{~d}, J_{\mathrm{HH}}=6.9 \mathrm{~Hz}, 3 \mathrm{H}\right), 1.16(\mathrm{~d}$, $\left.J_{\mathrm{HH}}=6.2 \mathrm{~Hz}, 3 \mathrm{H}\right), 1.02\left(\mathrm{~d}, J_{\mathrm{HH}}=6.9 \mathrm{~Hz}, 3 \mathrm{H}\right) ;{ }^{13} \mathrm{C}\left\{{ }^{1} \mathrm{H}\right\} \mathrm{NMR}\left(\mathrm{CD}_{3} \mathrm{CN}\right) \delta 194.6,145.6,144.3$, 133.1, 132.0, 126.7, 126.5, 72.8, 60.2, 50.3, 48.5, 40.6, 35.9, 31.1, 30.3, 29.9, 27.4, 26.6, 25.4, 24.0, 23.7, 23.5, 23.3, 22.6, 19.1, 18.4; MS(ESI) $\mathrm{m} / z 368[\mathrm{M}]^{+}$.

\section{Synthesis of Alkenylaldimine 6}

A solution of $n$-BuLi in hexanes $(3.6 \mathrm{~mL}, 1.6 \mathrm{M}, 5.8 \mathrm{mmol})$ was added dropwise to an $\mathrm{Et}_{2} \mathrm{O}$ solution $(20 \mathrm{~mL})$ of $\mathbf{5}^{20}(2.00 \mathrm{~g}, 5.8 \mathrm{mmol})$ at $-78^{\circ} \mathrm{C}$. After $15 \mathrm{~min}$, the mixture was warmed to $\mathrm{rt}$ and stirred for an additional $1 \mathrm{~h}$. The mixture was then cooled to $-78^{\circ} \mathrm{C}$, and 3-bromo-2methylpropene $(0.59 \mathrm{~mL}, 5.8 \mathrm{mmol})$ was slowly added. After $15 \mathrm{~min}$, the solution was warmed to $\mathrm{rt}$ and stirred for an additional $12 \mathrm{~h}$. Removal of the volatiles under vacuum and extraction with hexanes afforded 6 as a light yellow oil $(1.80 \mathrm{~g}, 97 \%):{ }^{1} \mathrm{H}$ NMR $\left(\mathrm{CDCl}_{3}\right) \delta 8.49(\mathrm{~s}, 1 \mathrm{H})$, 8.32-8.28 (m, 1H), 8.32-8.28 (m, 1H), 7.50-7.44 (m, 2H), 7.33-7.30 (m, 1H), 7.26-7.18 (m, $2 \mathrm{H}), 4.89(\mathrm{~s}, 1 \mathrm{H}), 4.48(\mathrm{~s}, 1 \mathrm{H}), 3.61(\mathrm{~s}, 2 \mathrm{H}), 3.07\left(\mathrm{sept}, J_{\mathrm{HH}}=6.8 \mathrm{~Hz}, 2 \mathrm{H}\right), 1.83(\mathrm{~s}, 3 \mathrm{H}), 1.24$ $\left(\mathrm{d}, J_{\mathrm{HH}}=6.8 \mathrm{~Hz}, 12 \mathrm{H}\right) ;{ }^{13} \mathrm{C}\left\{{ }^{1} \mathrm{H}\right\} \mathrm{NMR}\left(\mathrm{CDCl}_{3}\right) \delta 160.7,149.8,145.2,140.0,137.8,134.6$, $131.3,131.1,127.6,127.2,124.2,123.2,112.7,40.9,28.1,23.7,23.3$; $\mathrm{MS}(\mathrm{EI}) \mathrm{m} / \mathrm{z} 320[\mathrm{M}+$ $\mathrm{H}]^{+}$.

\section{Synthesis of Dihydroisoquinolinium 7}

Salt $\mathbf{7}$ was prepared from $\mathbf{6}$ following the same procedure as for the synthesis of $\mathbf{4 b}$. Precipitation of the residue from $\mathrm{Et}_{2} \mathrm{O}$ afforded 7 as a white powder $(95 \%): \mathrm{mp} 118{ }^{\circ} \mathrm{C} ;{ }^{1} \mathrm{H}$ $\operatorname{NMR}\left(\mathrm{CDCl}_{3}\right) \delta 9.98(\mathrm{~s}, 1 \mathrm{H}), 8.73\left(\mathrm{~d}, J_{\mathrm{HH}}=7.6 \mathrm{~Hz}, 1 \mathrm{H}\right), 7.82(\mathrm{br} \mathrm{dd}, 1 \mathrm{H}), 7.80-7.47(\mathrm{~m}, 3 \mathrm{H})$, $7.31\left(\mathrm{~d}, J_{\mathrm{HH}}=7.9 \mathrm{~Hz}, 2 \mathrm{H}\right), 3.54(\mathrm{~s}, 2 \mathrm{H}), 2.77\left(\mathrm{sept}, J_{\mathrm{HH}}=6.8 \mathrm{~Hz}, 2 \mathrm{H}\right), 1.43(\mathrm{~s}, 6 \mathrm{H}), 1.33(\mathrm{~d}$, $\left.J_{\mathrm{HH}}=6.8 \mathrm{~Hz}, 6 \mathrm{H}\right), 1.21\left(\mathrm{~d}, J_{\mathrm{HH}}=6.8 \mathrm{~Hz}, 6 \mathrm{H}\right) ;{ }^{13} \mathrm{C}\left\{{ }^{1} \mathrm{H}\right\} \mathrm{NMR}\left(\mathrm{CDCl}_{3}\right) \delta 172.0,143.8,139.5$, 138.1, 136.7, 135.0, 131.2, 129.0, 125.1, 125.0, 67.8, 40.7, 29.7, 26.2, 25.2, 23.0; MS-(HRESI) $m / z 320.2373[\mathrm{M}]^{+}($calcd 320.2378).

\section{Synthesis of Alkenylformamidines 9a-d}

To a THF solution $(40 \mathrm{~mL})$ of the corresponding formamidines $8(5.5 \mathrm{mmol})$ at $-78{ }^{\circ} \mathrm{C}$ was added a solution of $n$-BuLi in hexanes $(5.5 \mathrm{mmol})$. The mixture was stirred for $30 \mathrm{~min}$ and then was allowed to warm to rt and stirred for a further $12 \mathrm{~h}$. The mixture was again cooled to -78 ${ }^{\circ} \mathrm{C}$, and 3-bromopropene $(5.5 \mathrm{mmol})$ or 3-bromo-2-methylpropene $(5.5 \mathrm{mmol})$ was slowly added. The mixture was stirred for $30 \mathrm{~min}$ at $-78^{\circ} \mathrm{C}$ and then heated at $50^{\circ} \mathrm{C}$ for $12 \mathrm{~h}$. Removal of the volatiles under vacuum and extraction with hexanes afforded derivatives 9a-d.

Derivative 9a was obtained as a white solid (94\%): $\mathrm{mp} 62{ }^{\circ} \mathrm{C} ;{ }^{1} \mathrm{H}$ NMR $\left(\mathrm{CDCl}_{3}\right) \delta 7.37-6.99$ $(\mathrm{m}, 7 \mathrm{H}), 6.26-6.13(\mathrm{~m}, 1 \mathrm{H}), 5.19\left(\mathrm{~d}, J_{\mathrm{HH}}=5.5 \mathrm{~Hz}, 1 \mathrm{H}\right), 5.15(\mathrm{br} \mathrm{s}, 1 \mathrm{H}), 4.42\left(\mathrm{~d}, J_{\mathrm{HH}}=6.8\right.$ $\mathrm{Hz}, 2 \mathrm{H}), 3.33-3.18(\mathrm{~m}, 4 \mathrm{H}), 1.30\left(\mathrm{~d}, J_{\mathrm{HH}}=6.8 \mathrm{~Hz}, 6 \mathrm{H}\right), 1.22\left(\mathrm{~d}, J_{\mathrm{HH}}=6.8 \mathrm{~Hz}, 12 \mathrm{H}\right), 1.15(\mathrm{~d}$, 
$\left.J_{\mathrm{HH}}=6.8 \mathrm{~Hz}, 6 \mathrm{H}\right) ;{ }^{13} \mathrm{C}\left\{{ }^{1} \mathrm{H}\right\} \operatorname{NMR}\left(\mathrm{CDCl}_{3}\right) \delta 151.1,148.4,147.5,140.1,138.4,133.4,128.9$, 124.4, 122.8, 118.3, 53.0, 28.5, 28.0, 25.4, 24.4, 23.8; MS-(EI) $\mathrm{m} / z 405[\mathrm{M}+\mathrm{H}]^{+}$. Derivative 9b was obtained as a white solid (89\%): $\mathrm{mp} 73{ }^{\circ} \mathrm{C} ;{ }^{1} \mathrm{H} \mathrm{NMR}\left(\mathrm{CDCl}_{3}\right) \delta 7.34-6.96(\mathrm{~m}, 7 \mathrm{H})$, $4.84(\mathrm{~s}, 1 \mathrm{H}), 4.68(\mathrm{~s}, 1 \mathrm{H}), 4.41(\mathrm{~s}, 2 \mathrm{H}), 3.24(\mathrm{~m}, 4 \mathrm{H}), 1.98(\mathrm{~s}, 3 \mathrm{H}), 1.29\left(\mathrm{~d}, J_{\mathrm{HH}}=6.7 \mathrm{~Hz}, 6 \mathrm{H}\right)$, $1.20\left(\mathrm{~d}, J_{\mathrm{HH}}=6.8 \mathrm{~Hz}, 12 \mathrm{H}\right), 1.12\left(\mathrm{~d}, J_{\mathrm{HH}}=6.8 \mathrm{~Hz}, 6 \mathrm{H}\right) ;{ }^{13} \mathrm{C}\left\{{ }^{1} \mathrm{H}\right\} \mathrm{NMR}\left(\mathrm{CDCl}_{3}\right) \delta 152.2$, 148.4, 147.5, 141.5, 140.0, 138.7, 128.7, 124.4, 122.8, 115.8, 55.3, 28.4, 28.0, 25.6, 24.5, 23.8, 21.9; MS(EI) $m / z: 419[\mathrm{M}+\mathrm{H}]^{+}$. Derivative $9 \mathrm{c}$ was obtained as a white solid (91\%): $\mathrm{mp} 72^{\circ}$ $\mathrm{C} ;{ }^{1} \mathrm{H}$ NMR $\left(\mathrm{C}_{6} \mathrm{D}_{6}\right) \delta 6.91$ (br s, $\left.2 \mathrm{H}\right), 6.80($ br s, $1 \mathrm{H}), 6.69$ (br s, $\left.2 \mathrm{H}\right), 4.77$ (br s, 2H), 4.36 (br $\mathrm{s}, 2 \mathrm{H}), 2.28(\mathrm{~s}, 10 \mathrm{H}), 2.09(\mathrm{~s}, 8 \mathrm{H}), 1.98(\mathrm{~s}, 3 \mathrm{H}) ;{ }^{13} \mathrm{C}\left\{{ }^{1} \mathrm{H}\right\} \mathrm{NMR}\left(\mathrm{C}_{6} \mathrm{D}_{6}\right) \delta 153.4,148.8,143.4$, 140.6, 137.6, 137.4, 131.2, 130.0, 129.4, 115.1, 54.2, 22.6, 21.3, 21.2, 19.7, 18.8; MS(EI) $\mathrm{m} /$ $z 335[\mathrm{M}+\mathrm{H}]^{+}$. Derivative 9d was obtained as a yellow oil $(92 \%):{ }^{1} \mathrm{H} \mathrm{NMR}\left(\mathrm{CDCl}_{3}\right) \delta 7.89$ $(\mathrm{m}, 1 \mathrm{H}), 7.26(\mathrm{~m}, 1 \mathrm{H}), 7.00(\mathrm{~m}, 2 \mathrm{H}), 6.87(\mathrm{~m}, 3 \mathrm{H}), 4.87(\mathrm{~s}, 1 \mathrm{H}), 4.81(\mathrm{~s}, 1 \mathrm{H}), 4.63(\mathrm{~s}, 2 \mathrm{H})$, $1.82(\mathrm{~s}, 3 \mathrm{H}) ;{ }^{13} \mathrm{C}\left\{{ }^{1} \mathrm{H}\right\} \mathrm{NMR}\left(\mathrm{CDCl}_{3}\right) \delta 159.2\left(\mathrm{~d}, J_{\mathrm{CF}}=226.0 \mathrm{~Hz}\right), 156.4\left(\mathrm{dd}, J_{\mathrm{CF}}=244.1,6.0\right.$ $\mathrm{Hz}), 155.8,140.6,128.2\left(\mathrm{t}, J_{\mathrm{CF}}=8.8 \mathrm{~Hz}\right), 122.4\left(\mathrm{t}, J_{\mathrm{CF}}=9.6 \mathrm{~Hz}\right), 114.1,112.4\left(\mathrm{~d}, J_{\mathrm{CF}}=22.4\right.$ Hz), 111.5 (br s), 51.7, 20.0; MS(EI) $\mathrm{m} / z 323[\mathrm{M}+\mathrm{H}]^{+}$.

\section{Synthesis of Alkenylformamidinium 10a}

Following the procedure described for the synthesis of 3a, derivative 10a was obtained from 9a as a white powder (95\%). Crystals were grown from chloroform: mp $151{ }^{\circ} \mathrm{C} \mathrm{dec} ;{ }^{1} \mathrm{H}$ NMR $\left(\mathrm{CDCl}_{3}\right) \delta 14.0\left(\mathrm{~d}, J_{\mathrm{HH}}=12.0 \mathrm{~Hz}, 1 \mathrm{H}\right), 7.52-7.40(\mathrm{~m}, 1 \mathrm{H}), 7.36-7.19(\mathrm{~m}, 6 \mathrm{H}), 5.97(\mathrm{~m}, 1 \mathrm{H})$, $5.64\left(\mathrm{~d}, J_{\mathrm{HH}}=16.9 \mathrm{~Hz}, 1 \mathrm{H}\right), 5.38\left(\mathrm{~d}, J_{\mathrm{HH}}=10.0 \mathrm{~Hz}, 1 \mathrm{H}\right), 5.14\left(\mathrm{~d}, J_{\mathrm{HH}}=7.1 \mathrm{~Hz}, 2 \mathrm{H}\right), 3.27$ (sept, $\left.J_{\mathrm{HH}}=6.8 \mathrm{~Hz}, 2 \mathrm{H}\right), 3.00\left(\mathrm{sept}, J_{\mathrm{HH}}=6.8 \mathrm{~Hz}, 2 \mathrm{H}\right), 1.40\left(\mathrm{br} \mathrm{d}, J_{\mathrm{HH}}=6.8 \mathrm{~Hz}, 6 \mathrm{H}\right), 1.32$ $\left(\mathrm{d}, J_{\mathrm{HH}}=6.8 \mathrm{~Hz}, 6 \mathrm{H}\right), 1.22\left(\mathrm{br} \mathrm{d}, J_{\mathrm{HH}}=6.8 \mathrm{~Hz}, 6 \mathrm{H}\right), 1.15\left(\mathrm{~d}, J_{\mathrm{HH}}=6.8 \mathrm{~Hz}, 6 \mathrm{H}\right) ;{ }^{13} \mathrm{C}\left\{{ }^{1} \mathrm{H}\right\}$ NMR $\left(\mathrm{CDCl}_{3}\right) \delta 155.5,146.0,145.8,135.7,132.2,131.0,129.9,128.0,125.3,124.2,123.8$, 57.2, 29.0, 25.5, 24.4, 24.0, 23.6; MS(HR-ESI) $\mathrm{m} / 2$ 405.3266 [M] ${ }^{+}$(calcd 405.3264).

\section{Synthesis of $\mathrm{NHC}, \mathrm{H}^{+} \mathrm{s}$ 11a-d from 9a-d}

Following the procedure described for the synthesis of $\mathbf{4 b}$, but heating at the temperature and during time indicated hereafter, derivatives 11a-d were isolated as white solids after washing with toluene and ether. 11a: $36 \mathrm{~h}$ at $135^{\circ} \mathrm{C} ; 83 \%$ yield; mp $204{ }^{\circ} \mathrm{C} ;{ }^{1} \mathrm{H}$ NMR $\left(\mathrm{CD}_{3} \mathrm{CN} /\right.$ $\left.\mathrm{CDCl}_{3}, 20: 1\right) \delta 9.39(\mathrm{~s}, 1 \mathrm{H}), 7.53(\mathrm{~m}, 2 \mathrm{H}), 7.56(\mathrm{~m}, 4 \mathrm{H}), 4.88(\mathrm{~m}, 1 \mathrm{H}), 4.63\left(\mathrm{t}, J_{\mathrm{HH}}=11.8 \mathrm{~Hz}\right.$, $1 \mathrm{H}), 4.01\left(\mathrm{dd}, J_{\mathrm{HH}}=11.8,10.2 \mathrm{~Hz}, 1 \mathrm{H}\right), 3.04(\mathrm{~m}, 4 \mathrm{H}), 1.45\left(\mathrm{~d}, J_{\mathrm{HH}}=6.5 \mathrm{~Hz}, 3 \mathrm{H}\right), 1.36(\mathrm{~m}$, $12 \mathrm{H}), 1.26-1.21(\mathrm{~m}, 12 \mathrm{H}) ;{ }^{13} \mathrm{C}\left\{{ }^{1} \mathrm{H}\right\} \mathrm{NMR}\left(\mathrm{CD}_{3} \mathrm{CN} / \mathrm{CDCl}_{3}, 20: 1\right) \delta 161.5,148.4,147.7,147.6$, 147.3, 132.4, 130.7, 128.7, 126.2, 126.0, 63.5, 61.2, 30.0, 29.9, 26.2, 25.6, 24.1, 24.0, 23.5, 18.5; MS(FAB) $\mathrm{m} / z 405[\mathrm{M}]^{+}$. 11b: $24 \mathrm{~h}$ at $110{ }^{\circ} \mathrm{C} ; 80 \%$ yield; $\mathrm{mp} 212{ }^{\circ} \mathrm{C} ;{ }^{1} \mathrm{H}$ NMR $\left(\mathrm{CD}_{3} \mathrm{CN}\right)$ $\delta 10.45(\mathrm{~s}, 1 \mathrm{H}), 7.58(\mathrm{~m}, 2 \mathrm{H}), 7.43\left(\mathrm{t}, J_{\mathrm{HH}}=7.7 \mathrm{~Hz}, 4 \mathrm{H}\right), 4.23(\mathrm{~s}, 2 \mathrm{H}), 3.16(\mathrm{~m}, 4 \mathrm{H}), 1.59(\mathrm{~s}$, $6 \mathrm{H}), 1.46\left(\mathrm{~d}, J_{\mathrm{HH}}=6.7 \mathrm{~Hz}, 12 \mathrm{H}\right), 1.40\left(\mathrm{~d}, J_{\mathrm{HH}}=6.8 \mathrm{~Hz}, 6 \mathrm{H}\right), 1.30\left(\mathrm{~d}, J_{\mathrm{HH}}=6.8 \mathrm{~Hz}\right.$, $6 \mathrm{H}) ;{ }^{13} \mathrm{C}\left\{{ }^{1} \mathrm{H}\right\} \mathrm{NMR}\left(\mathrm{CD}_{3} \mathrm{CN}\right) \delta 161.9,149.9,148.1,132.8,131.5,127.5,126.6,126.4,72.2$, 67.0, 31.1, 30.6, 27.8, 27.4, 26.2, 24.4, 23.4; $\mathrm{MS}(\mathrm{FAB}) \mathrm{m} / \mathrm{z} 419[\mathrm{M}]^{+} .11 \mathrm{c}: 12 \mathrm{~h}$ at $135^{\circ} \mathrm{C}$; $78 \%$ yield; mp $166{ }^{\circ} \mathrm{C} ;{ }^{1} \mathrm{H}$ NMR $\left(\mathrm{CDCl}_{3}\right) \delta 9.76(\mathrm{~s}, 1 \mathrm{H}), 7.01(\mathrm{~s}, 2 \mathrm{H}), 6.98(\mathrm{~s}, 2 \mathrm{H}), 2.44(\mathrm{~s}$, $6 \mathrm{H}), 2.42(\mathrm{~s}, 6 \mathrm{H}), 2.31(\mathrm{~s}, 3 \mathrm{H}), 2.30(\mathrm{~s}, 3 \mathrm{H}), 1.67(\mathrm{~s}, 6 \mathrm{H}) ;{ }^{13} \mathrm{C}\left\{{ }^{1} \mathrm{H}\right\} \mathrm{NMR}\left(\mathrm{CDCl}_{3}\right) \delta 159.4$, 140.5, 140.3, 137.0, 135.0, 133.8, 130.6, 130.2, 127.4, 71.5, 64.0, 27.2, 21.2, 21.1, 20.1, 18.3; MS-(FAB) $\mathrm{m} / \mathrm{z} 335[\mathrm{M}]^{+} .11 \mathrm{~d}: 12 \mathrm{~h}$ at $135{ }^{\circ} \mathrm{C} ; 79 \%$ yield; mp $186{ }^{\circ} \mathrm{C} ;{ }^{1} \mathrm{H}$ NMR $\left(\mathrm{CD}_{3} \mathrm{CN}\right) \delta$ $9.54(\mathrm{~s}, 1 \mathrm{H}), 7.70(\mathrm{~m}, 1 \mathrm{H}), 7.59(\mathrm{~m} \mathrm{1H}), 7.30(\mathrm{~m}, 4 \mathrm{H}), 4.45(\mathrm{~s}, 2 \mathrm{H}), 1.60(\mathrm{~s}, 6 \mathrm{H}) ;{ }^{13} \mathrm{C}\left\{{ }^{1} \mathrm{H}\right\}$ $\operatorname{NMR}\left(\mathrm{CD}_{3} \mathrm{CN}\right) \delta 162.1,160.2\left(\mathrm{dd}, J_{\mathrm{CF}}=249.7,6.0 \mathrm{~Hz}\right), 158.1\left(\mathrm{dd}, J_{\mathrm{CF}}=248.2,6.0 \mathrm{~Hz}\right)$, $134.8\left(\mathrm{t}, J_{\mathrm{CF}}=10.3 \mathrm{~Hz}\right), 132.9\left(\mathrm{t}, J_{\mathrm{CF}}=10.1 \mathrm{~Hz}\right), 114.0\left(\mathrm{dd}, J_{\mathrm{CF}}=7.4,3.2 \mathrm{~Hz}\right), 113.8,(\mathrm{dd}$, $\left.J_{\mathrm{CF}}=6.8,3.3 \mathrm{~Hz}\right), 72.9,64.0,25.4$; MS(HR-ESI) $\mathrm{m} / z 323.1178[\mathrm{M}]^{+}($calcd 323.1171).

\section{Synthesis of $\mathrm{NHC}, \mathrm{H}^{+}$s $11 \mathrm{a}, \mathrm{b}$ from $8 \mathrm{a}$}

A tube sealed by a Teflon stopcock was loaded with $8 \mathbf{a}(2.00 \mathrm{~g}, 5.5 \mathrm{mmol})$, toluene $(20 \mathrm{~mL})$, and 3-bromopropene $(0.474 \mathrm{~mL}, 5.5 \mathrm{mmol})$ or 3-bromo-2-methylpropene $(0.54 \mathrm{~mL}, 5.5$ 
mmol). Heating for $36 \mathrm{~h}$ at $135^{\circ} \mathrm{C}$ or $24 \mathrm{~h}$ at $110{ }^{\circ} \mathrm{C}$ afforded 11a and $11 \mathrm{~b}$, respectively, as white solids after removal of the volatiles in vacuo and washing with toluene, 11a: $1.76 \mathrm{~g}, 66 \%$; mp200 ${ }^{\circ} \mathrm{C}$. 11b: $1.92 \mathrm{~g}, 70 \%$; $\mathrm{mp} 204{ }^{\circ} \mathrm{C}$.

\section{Synthesis of 13}

Following the procedure for 9a, amidine $\mathbf{1 2}^{21}(1.17 \mathrm{~g}, 2.7 \mathrm{mmol})$ was converted to its corresponding alkenyl derivative using 1 equiv of $n$ - $\mathrm{BuLi}$ and 3-bromo-2-methylpropene. The alkenylamidine was obtained as a white solid $(1.25 \mathrm{~g}, 95 \%)$ : $\mathrm{mp} 90{ }^{\circ} \mathrm{C} ;{ }^{1} \mathrm{H} \mathrm{NMR}\left(\mathrm{CDCl}_{3}\right) \delta$ $7.42-6.84(\mathrm{~m}, 11 \mathrm{H}), 5.25(\mathrm{~s}, 1 \mathrm{H}), 4.98(\mathrm{~s}, 1 \mathrm{H}), 4.39(\mathrm{~s}, 2 \mathrm{H}), 3.69\left(\mathrm{sept}, J_{\mathrm{HH}}=6.6 \mathrm{~Hz}, 2 \mathrm{H}\right)$, $3.17\left(\mathrm{sept}, J_{\mathrm{HH}}=6.6 \mathrm{~Hz}, 2 \mathrm{H}\right), 1.90(\mathrm{~s}, 3 \mathrm{H}), 1.26\left(\mathrm{~d}, J_{\mathrm{HH}}=6.6 \mathrm{~Hz}, 6 \mathrm{H}\right), 1.19(2 \mathrm{x}$ overlapping $\left.\mathrm{d}, J_{\mathrm{HH}}=6.6 \mathrm{~Hz}, 12 \mathrm{H}\right), 0.98\left(\mathrm{~d}, J_{\mathrm{HH}}=6.6 \mathrm{~Hz}, 6 \mathrm{H}\right) ;{ }^{13} \mathrm{C}\left\{{ }^{1} \mathrm{H}\right\} \mathrm{NMR}\left(\mathrm{CDCl}_{3}\right) \delta 156.7,147.5$, $145.4,142.0,141.4,138.0,133.6,128.9,128.4,128.0,127.0,124.4,122.6,121.7,110.5,58.6$, 28.5, 28.4, 26.6, 24.8, 23.2, 22.3, 22.0; MS(FAB) $m / z 495[\mathrm{M}+\mathrm{H}]^{+}$. Following the procedure described for the synthesis of $\mathbf{4 b , 1 3}$ was obtained from 12 in $85 \%$ yield: $\mathrm{mp} 181{ }^{\circ} \mathrm{C} ;{ }^{1} \mathrm{H}$ NMR $\left(\mathrm{CDCl}_{3}\right) \delta 7.40-7.24(\mathrm{~m}, 3 \mathrm{H}), 7.17-7.03(\mathrm{~m}, 6 \mathrm{H}), 6.92(\mathrm{~m}, 2 \mathrm{H}), 4.45(\mathrm{~s}, 2 \mathrm{H}), 2.83$ (sept, $J_{\mathrm{HH}}$ $=6.6 \mathrm{~Hz}, 4 \mathrm{H}), 1.71(\mathrm{~s}, 6 \mathrm{H}), 1.27\left(\mathrm{~d}, J_{\mathrm{HH}}=6.6 \mathrm{~Hz}, 6 \mathrm{H}\right), 1.25\left(\mathrm{~d}, J_{\mathrm{HH}}=6.6 \mathrm{~Hz}, 6 \mathrm{H}\right), 0.86(\mathrm{~d}$, $\left.J_{\mathrm{HH}}=6.6 \mathrm{~Hz}, 6 \mathrm{H}\right), 0.70\left(\mathrm{~d}, J_{\mathrm{HH}}=6.6 \mathrm{~Hz}, 6 \mathrm{H}\right) ;{ }^{13} \mathrm{C}\left\{{ }^{1} \mathrm{H}\right\} \mathrm{NMR}\left(\mathrm{CDCl}_{3}\right) \delta 165.8,147.0,144.8$, 134.3, 131.4, 131.2, 129.5, 128.8, 127.9, 126.2, 125.5, 121.5, 70.9, 66.5, 29.4, 29.1, 26.3, 25.6, 25.1, 24.5, 23.1; $\mathrm{MS}(\mathrm{FAB}) \mathrm{mlz} 495[\mathrm{M}]^{+}$.

\section{Synthesis of Alkenylurea 14}

To a solution of lithium (2,6-diisopropylphenyl)amide 22 (1.5 g, $8.2 \mathrm{mmol})$ in $^{2} \mathrm{Et}_{2} \mathrm{O}(20 \mathrm{~mL})$ at $\mathrm{rt}$ was added dropwise 3-bromo-2-methylpropene $(0.91 \mathrm{~mL}, 1.22 \mathrm{~g}, 9.0 \mathrm{mmol})$. The solution was stirred for $2 \mathrm{~h}$, and then the volatiles were removed under vacuum and the residue was extracted with hexanes and filtered. Removal of volatiles from the filtrate under vacuum provided (2,6-diisopropylphenyl)(2-methylallyl)amine as a colorless liquid: yield (1.71 g, 90\%); ${ }^{1} \mathrm{H}$ NMR $\left(\mathrm{CDCl}_{3}\right) \delta 7.12(\mathrm{~m}, 3 \mathrm{H}), 5.15(\mathrm{~s}, 1 \mathrm{H}), 4.94(\mathrm{~s}, 1 \mathrm{H}), 3.42\left(\mathrm{~d}, J_{\mathrm{HH}}=7.6 \mathrm{~Hz}, 2 \mathrm{H}\right)$, 3.29 (sept, $\left.J_{\mathrm{HH}}=6.8 \mathrm{~Hz}, 2 \mathrm{H}\right), 2.97\left(\mathrm{br} \mathrm{t}, J_{\mathrm{HH}}=7.4 \mathrm{~Hz}, 1 \mathrm{H}\right), 1.89(\mathrm{~s}, 3 \mathrm{H}), 1.27\left(\mathrm{~d}, J_{\mathrm{HH}}=6.8\right.$ $\mathrm{Hz}, 12 \mathrm{H}$ ). ${ }^{13} \mathrm{C}$ NMR (neat) $\delta 144.1,143.3,142.6,124.2,123.4,110.8,58.1,27.7,24.3,20.8$.

A solution of $n$ - $\mathrm{BuLi}$ in hexanes $\left(5.4 \mathrm{~mL}, 1.6 \mathrm{M}, 8.6 \mathrm{mmol}\right.$ ) was added to an $\mathrm{Et}_{2} \mathrm{O}$ solution (40 $\mathrm{mL}$ ) of (2,6-diisopropylphenyl)(2-methylallyl)amine $(2.00 \mathrm{~g}, 8.6 \mathrm{mmol})$ at $-78{ }^{\circ} \mathrm{C}$. After 30 $\mathrm{min}$, the mixture was warmed to $\mathrm{rt}$ and stirred for $3 \mathrm{~h}$. The mixture was then cooled to $-78^{\circ}$ $\mathrm{C}$, and 2,6-diisopropylphenyl isocyanate $(1.85 \mathrm{~mL}, 8.6 \mathrm{mmol})$ was added dropwise. The mixture was warmed to rt and stirred for $12 \mathrm{~h}$, over which time a white precipitate formed. The mixture was cooled in an ice bath, and $\mathrm{H}_{2} \mathrm{O}(40 \mathrm{~mL})$ was added slowly. Extraction with $\mathrm{Et}_{2} \mathrm{O}$, drying over $\mathrm{MgSO}_{4}$, and removal of the volatiles under vacuum afforded 14 as an off-white crystalline solid $(3.34 \mathrm{~g}, 89 \%)$ : $\mathrm{mp} 147{ }^{\circ} \mathrm{C} ;{ }^{1} \mathrm{H} \mathrm{NMR}\left(\mathrm{CDCl}_{3}\right) \delta 7.38\left(\mathrm{t}, J_{\mathrm{HH}}=7.4 \mathrm{~Hz}, 1 \mathrm{H}\right)$, $7.27\left(\mathrm{~d}, J_{\mathrm{HH}}=7.4 \mathrm{~Hz}, 2 \mathrm{H}\right), 7.21\left(\mathrm{t}, J_{\mathrm{HH}}=7.4 \mathrm{~Hz}, 1 \mathrm{H}\right), 7.12\left(\mathrm{~d}, J_{\mathrm{HH}}=7.4 \mathrm{~Hz}, 2 \mathrm{H}\right), 5.40(\mathrm{~s}$, $1 \mathrm{H}), 4.81(\mathrm{~s}, 1 \mathrm{H}), 4.70(\mathrm{~s}, 1 \mathrm{H}), 4.22(\mathrm{~s}, 2 \mathrm{H}), 3.30\left(\mathrm{sept}, J_{\mathrm{HH}}=6.8 \mathrm{~Hz}, 2 \mathrm{H}\right), 3.13\left(\mathrm{sept}, J_{\mathrm{HH}}=\right.$ $6.8 \mathrm{~Hz}, 2 \mathrm{H}), 1.93(\mathrm{~s}, 3 \mathrm{H}), 1.31\left(\mathrm{~d}, J_{\mathrm{HH}}=6.8 \mathrm{~Hz}, 6 \mathrm{H}\right), 1.28\left(\mathrm{~d}, J_{\mathrm{HH}}=6.8 \mathrm{~Hz}, 6 \mathrm{H}\right), 1.17(\mathrm{~d}$, $\left.J_{\mathrm{HH}}=6.8 \mathrm{~Hz}, 12 \mathrm{H}\right) ;{ }^{13} \mathrm{C}\left\{{ }^{1} \mathrm{H}\right\} \mathrm{NMR}\left(\mathrm{CDCl}_{3}\right) \delta 156.8,148.5,146.8,142.9,136.8,132.0,129.3$, $127.8,125.3,123.5,114.2,57.3,28.6,26.2,24.6,24.2,21.2$.

\section{Synthesis of $\boldsymbol{C}$-chloro Imidazolinium Salt 15}

To a toluene solution $(10 \mathrm{~mL})$ of alkenylurea $14(1.00 \mathrm{~g}, 2.3 \mathrm{mmol})$ in a sealable tube fitted with a Teflon stopcock at $-78{ }^{\circ} \mathrm{C}$ was added a toluene solution of phosgene $(1.26 \mathrm{~mL}, 2.0 \mathrm{M}$, $2.5 \mathrm{mmol})$. After $10 \mathrm{~min}$ at $-78^{\circ} \mathrm{C}$, the mixture was warmed to rt and stirred for $2 \mathrm{~h}$. The mixture was then sealed and heated at $80^{\circ} \mathrm{C}$ for $24 \mathrm{~h}$. After removal of the volatiles in vacuo and precipitation of the product from $\mathrm{Et}_{2} \mathrm{O}(20 \mathrm{~mL}), \mathbf{1 5}$ was obtained as a white solid $(0.78 \mathrm{~g}$, 78\%): $\mathrm{mp} 142{ }^{\circ} \mathrm{C} ;{ }^{1} \mathrm{H}$ NMR $\left(\mathrm{CDCl}_{3}\right) \delta 7.48\left(\mathrm{t}, J_{\mathrm{HH}}=7.7 \mathrm{~Hz}, 1 \mathrm{H}\right), 7.29(\mathrm{~m}, 3 \mathrm{H}), 7.12(\mathrm{~d}$, $\left.J_{\mathrm{HH}}=7.7 \mathrm{~Hz}, 2 \mathrm{H}\right), 3.90(\mathrm{~s}, 2 \mathrm{H}), 2.99\left(\mathrm{sept}, J_{\mathrm{HH}}=6.8 \mathrm{~Hz}, 4 \mathrm{H}\right), 1.66(\mathrm{~s}, 6 \mathrm{H}), 1.48\left(\mathrm{~d}, J_{\mathrm{HH}}=\right.$ 
$6.8 \mathrm{~Hz}, 6 \mathrm{H}), 1.31\left(\mathrm{~d}, J_{\mathrm{HH}}=6.8 \mathrm{~Hz}, 6 \mathrm{H}\right), 1.20\left(\mathrm{~d}, J_{\mathrm{HH}}=6.8 \mathrm{~Hz}, 6 \mathrm{H}\right), 1.14\left(\mathrm{~d}, J_{\mathrm{HH}}=6.8 \mathrm{~Hz}\right.$, $6 \mathrm{H}) ;{ }^{13} \mathrm{C}\left\{{ }^{1} \mathrm{H}\right\} \mathrm{NMR}\left(\mathrm{CDCl}_{3}\right) \delta 158.2,148.6,145.8,132.0,129.4,126.6,125.7,123.8,88.7$, 64.1, 29.4 28.8, 27.7, 25.7, 24.2, 23.8; MS-(FAB) $\mathrm{m} / z 435[\mathrm{M}-\mathrm{Cl}+(\mathrm{OH})]^{+}$.

\section{Synthesis of Alkenylamides $16 a, b$}

A solution of $n$-BuLi in hexanes $(6.3 \mathrm{~mL}, 1.6 \mathrm{M}, 10.2 \mathrm{mmol})$ was added to an $\mathrm{Et}_{2} \mathrm{O}$ solution $(20 \mathrm{~mL})$ of 1-bromo-2-isopropenylbenzene ${ }^{23}(2.00 \mathrm{~g}, 10.2 \mathrm{mmol})$ at $-78^{\circ} \mathrm{C}$. After $30 \mathrm{~min}$, the mixture was warmed to $\mathrm{rt}$ and stirred for an additional $1 \mathrm{~h}$. The mixture was then cooled to -78 ${ }^{\circ} \mathrm{C}$, and 2,6-diisopropylphenylisocyanate $(2.17 \mathrm{~mL}, 10.2 \mathrm{mmol})$ or tert-butyl isocyanate (1.17 $\mathrm{mL}, 10.2 \mathrm{mmol}$ ) was slowly added. The mixture was allowed to warm to $\mathrm{rt}$ and stirred for 12 $\mathrm{h}$ while a white precipitate formed. After the mixture was cooled with an ice bath, $\mathrm{H}_{2} \mathrm{O}(20$ $\mathrm{mL}$ ) was added slowly. Extraction with $\mathrm{Et}_{2} \mathrm{O}$, drying over $\mathrm{MgSO}_{4}$, and removal of the volatiles under vacuum afforded 16a,b as off-white crystalline solids. 16a: $2.66 \mathrm{~g}, 81 \%$ yield; $\mathrm{mp} 115^{\circ}$ $\mathrm{C} ;{ }^{1} \mathrm{H} \mathrm{NMR}\left(\mathrm{CDCl}_{3}\right) \delta 7.83\left(\mathrm{dd}, J_{\mathrm{HH}}=1.9,7.4 \mathrm{~Hz}, 1 \mathrm{H}\right), 7.48-7.21(\mathrm{~m}, 6 \mathrm{H}), 5.30(\mathrm{~s}, 1 \mathrm{H}), 5.16$ $(\mathrm{s}, 1 \mathrm{H}), 3.21\left(\mathrm{sept}, J_{\mathrm{HH}}=6.8 \mathrm{~Hz}, 2 \mathrm{H}\right), 2.24(\mathrm{~s}, 3 \mathrm{H}), 1.25\left(\mathrm{~d}, J_{\mathrm{HH}}=6.8 \mathrm{~Hz}, 12 \mathrm{H}\right) ;{ }^{13} \mathrm{C}\left\{{ }^{1} \mathrm{H}\right\}$ NMR $\left(\mathrm{CDCl}_{3}\right) \delta 168.5,146.8,146.5,142.5,134.3,131.2,130.6,129.2,128.8,128.6,127.7$, 123.8, 116.1, 29.0, 25.4, 24.0; MS(EI) $\mathrm{m} / \mathrm{z} 322[\mathrm{M}+\mathrm{H}]^{+}$. 16b: $1.93 \mathrm{~g}, 87 \%$ yield; ${ }^{1} \mathrm{H}$ NMR $\left(\mathrm{CDCl}_{3}\right) \delta 7.66\left(\mathrm{dd}, J_{\mathrm{HH}}=1.8,7.2 \mathrm{~Hz}, 1 \mathrm{H}\right), 7.36(\mathrm{~m}, 2 \mathrm{H}), 7.20\left(\mathrm{dd}, J_{\mathrm{HH}}=1.5,7.2 \mathrm{~Hz}, 1 \mathrm{H}\right)$, $6.00(\mathrm{bs}, 1 \mathrm{H}), 5.24(\mathrm{~s}, 1 \mathrm{H}), 5.10(\mathrm{~s}, 1 \mathrm{H}), 2.12(\mathrm{~s}, 3 \mathrm{H}), 1.44(\mathrm{~s}, 9 \mathrm{H}) ;{ }^{13} \mathrm{C}\left\{{ }^{1} \mathrm{H}\right\} \operatorname{NMR}\left(\mathrm{CDCl}_{3}\right)$ $\delta$ 168.0, 146.0, 141.1, 135.0, 130.2, 129.0, 128.7, 127.7, 115.9, 51.8, 28.7, 24.7.

\section{Synthesis of Cyclic $C$-Chloro Iminium Salt 17a,b}

Following the procedure used for $\mathbf{1 5}$ and heating as indicated hereafter, $\mathbf{1 7} \mathbf{a}, \mathbf{b}$ were obtained as off-white solids. 17a: $80{ }^{\circ} \mathrm{C}$ for $24 \mathrm{~h}, 86 \%$ yield; mp $130{ }^{\circ} \mathrm{C} ;{ }^{1} \mathrm{H}$ NMR $\left(\mathrm{CD}_{3} \mathrm{CN}\right) \delta 9.49(\mathrm{~d}$, $\left.J_{\mathrm{HH}}=7.9 \mathrm{~Hz}, 1 \mathrm{H}\right), 7.95\left(\mathrm{t}, J_{\mathrm{HH}}=7.6 \mathrm{~Hz}, 1 \mathrm{H}\right), 7.76(\mathrm{~m}, 2 \mathrm{H}), 7.48\left(\mathrm{t}, J_{\mathrm{HH}}=7.7 \mathrm{~Hz}, 1 \mathrm{H}\right), 7.36$ $\left(\mathrm{d}, J_{\mathrm{HH}}=7.9 \mathrm{~Hz}, 2 \mathrm{H}\right), 3.08\left(\mathrm{sept}, J_{\mathrm{HH}}=6.8 \mathrm{~Hz}, 2 \mathrm{H}\right), 1.72(\mathrm{~s}, 6 \mathrm{H}), 1.24\left(\mathrm{~d}, J_{\mathrm{HH}}=6.8 \mathrm{~Hz}, 6 \mathrm{H}\right)$, $1.16\left(\mathrm{~d}, J_{\mathrm{HH}}=6.8 \mathrm{~Hz}, 6 \mathrm{H}\right) ;{ }^{13} \mathrm{C}-\left\{{ }^{1} \mathrm{H}\right\} \mathrm{NMR}\left(\mathrm{CDCl}_{3}\right) \delta 171.6,155.2,145.6,138.0,131.2,130.8$, 129.2, 125.2, 123.9, 122.6, 100.4, 29.7, 26.7, 24.3, 23.6. MS-(HR-ESI) $\mathrm{m} / z$ 322.2174 [M - Cl $+\mathrm{OH}]^{+}($calcd 322.2170$) .17 \mathrm{~b}: 80{ }^{\circ} \mathrm{C}$ for $24 \mathrm{~h}, 78 \%$ yield; mp $154{ }^{\circ} \mathrm{C} ;{ }^{1} \mathrm{H} \mathrm{NMR}\left(\mathrm{CDCl}_{3}\right) \delta$ $9.77\left(\mathrm{~d}, J_{\mathrm{HH}}=7.9 \mathrm{~Hz}, 1 \mathrm{H}\right), 7.76\left(\mathrm{t}, J_{\mathrm{HH}}=7.5 \mathrm{~Hz}, 1 \mathrm{H}\right), 7.64\left(\mathrm{t}, J_{\mathrm{HH}}=7.7 \mathrm{~Hz}, 1 \mathrm{H}\right), 7.39(\mathrm{~d}$, $\left.J_{\mathrm{HH}}=7.7 \mathrm{~Hz}, 1 \mathrm{H}\right), 1.79(\mathrm{~s}, 6 \mathrm{H}), 1.67(\mathrm{~s}, 9 \mathrm{H}) ;{ }^{13} \mathrm{C}\left\{{ }^{1} \mathrm{H}\right\} \operatorname{NMR}\left(\mathrm{CDCl}_{3}\right) \delta 170.3,152.1,136.2$, $130.4,130.0,120.4,120.4,98.4,58.3,28.5,26.9 ; \mathrm{MS}(\mathrm{HR}-\mathrm{ESI}) \mathrm{m} / 2.218 .1543[\mathrm{M}-\mathrm{Cl}+$ $\mathrm{OH}]^{+}($calcd 218.1539).

\section{Palladium Complex 18a}

A $\mathrm{CH}_{2} \mathrm{Cl}_{2}(15 \mathrm{~mL})$ solution of $17 \mathrm{a}(0.10 \mathrm{~g}, 0.27 \mathrm{mmol})$ and tetrakis(triphenylphosphine) palladium $(0.31 \mathrm{~g}, 0.27 \mathrm{mmol})$ was heated under reflux for $24 \mathrm{~h}$. The solvent was removed under vacuum, then repeated extraction with boiling hexane to remove the liberated triphenylphosphine provided complex 18a as a yellow solid $(0.11 \mathrm{~g}, 55 \%):{ }^{1} \mathrm{H} \mathrm{NMR}\left(\mathrm{CDCl}_{3}\right)$ $\delta 7.67-7.19(\mathrm{~m}, 22 \mathrm{H}), 3.09\left(\mathrm{sept}, J_{\mathrm{HH}}=6.9 \mathrm{~Hz}, 2 \mathrm{H}\right), 1.61(\mathrm{~s}, 6 \mathrm{H}), 1.21\left(\mathrm{~d}, J_{\mathrm{HH}}=6.5 \mathrm{~Hz}\right.$, $12 \mathrm{H}) ;{ }^{13} \mathrm{C}\left\{{ }^{1} \mathrm{H}\right\} \mathrm{NMR}\left(\mathrm{CDCl}_{3}\right) \delta 221.1\left(\mathrm{~d}, J_{\mathrm{PC}}=175 \mathrm{~Hz}\right), 149.1(\mathrm{br}), 148.1,134.8\left(\mathrm{~d}, J_{\mathrm{PC}}=\right.$ $11.0 \mathrm{~Hz}), 133.7,132.9,130.6\left(\mathrm{~d}, J_{\mathrm{PC}}=40.5 \mathrm{~Hz}\right), 130.1,129.7,128.6,128.0\left(\mathrm{~d}, J_{\mathrm{PC}}=9.9 \mathrm{~Hz}\right)$, $125.7,120.0,84.3\left(\mathrm{~d}, J_{\mathrm{PC}}=7.9 \mathrm{~Hz}\right), 29.5,29.0,26.5,24.4 ;{ }^{13} \mathrm{C}$ NMR resonances corresponding to the aromatic quaternary carbons of the fused ring system could not be located; ${ }^{31} \mathrm{P}$ NMR $\left(\mathrm{CD}_{3} \mathrm{CN}\right) \delta+15.3$; MS(HR-ESI) $\mathrm{m} / z 778.1141[\mathrm{M}+\mathrm{Cl}]^{+}($calcd 778.1155$)$.

\section{Labeling Experiment: Synthesis of Cyclic Iminium Salt $\mathbf{4}_{\mathbf{D}}$}

In a sealable vessel fitted with a Teflon stopcock was bubbled deuterium chloride gas through a solution of alkenyl imine $2 \mathrm{c}(0.34 \mathrm{~g}, 1.0 \mathrm{mmol})$ in toluene for $1 \mathrm{~min}$. The vessel was then sealed and heated to $110^{\circ} \mathrm{C}$ for $16 \mathrm{~h}$. After the mixture was cooled to rt, the volatiles were remove under vacuum and the residue was extracted with $\mathrm{CD}_{3} \mathrm{CN}$ and transferred to an NMR 
tube: ${ }^{1} \mathrm{H}$ NMR $\left(\mathrm{CD}_{3} \mathrm{CN}\right) \delta 9.85(\mathrm{~s}, 1 \mathrm{H}, \mathrm{CH}=\mathrm{N}), 7.57\left(\mathrm{t}, J_{\mathrm{HH}}=7.79 \mathrm{~Hz}, 1 \mathrm{H}, \mathrm{H}_{\mathrm{ar}}\right), 7.43(\mathrm{~m}, 2 \mathrm{H}$, $\left.\mathrm{H}_{\mathrm{ar}}\right), 4.69(\mathrm{~m}, 1 \mathrm{H}, \mathrm{N}-\mathrm{CH}), 2.81-2.57\left(\mathrm{~m}, 3 \mathrm{H}, 2 \mathrm{H}\right.$ from $\mathrm{CH}_{i-\mathrm{Pr}}$ plus $1 \mathrm{H}$ from endocyclic $\left.\mathrm{CH}_{2}\right), 2.07-1.92\left(\mathrm{~m}, 3 \mathrm{H}\right.$, includes $1 \mathrm{H}$ from endocyclic $\left.\mathrm{CH}_{2}\right), 1.81(\mathrm{~m}, 4 \mathrm{H}), 1.67-1.46(\mathrm{~m}, 5 \mathrm{H}$, includes $\mathrm{CHD}_{\mathrm{Et}}$ ), $1.30-1.16$ (4 overlapping d, $\left.12 \mathrm{H}, \mathrm{CH}_{3}{ }^{i \mathrm{Pr}}\right), 0.85\left(\mathrm{~d}, J_{\mathrm{HH}}=6.48 \mathrm{~Hz}, 3 \mathrm{H}\right.$, $\left.\mathrm{CH}_{3-\mathrm{Et}}\right) ;{ }^{2} \mathrm{H}$ NMR $\left(\mathrm{CD}_{3} \mathrm{CN}\right) \delta 10.49$ (br, DCl ${ }_{2}$-counterion), $1.60\left(\mathrm{br}, \mathrm{CHD}_{\mathrm{Et}}\right) ;{ }^{13} \mathrm{C}\left\{{ }^{1} \mathrm{H}\right\}$ NMR $\left(\mathrm{CD}_{3} \mathrm{CN}\right) \delta 192.8(\mathrm{~N}=\mathrm{CH}), 144.6,143.4\left(\mathrm{C}_{\mathrm{ar}}{ }^{O}\right.$ rthO-DIPP $), 132.4\left(\mathrm{C}_{\mathrm{ar}}\right.$ Para-DIPP $), 131.5$ $\left(\mathrm{C}_{\mathrm{ar}}{ }^{\text {ipso-DIPP }}\right), 125.8125 .7\left(\mathrm{C}_{\mathrm{ar}}{ }^{\text {meta-DIPP }}\right), 78.1(\mathrm{~N}-\mathrm{CH}), 54.4(\mathrm{C}), 38.0$ (endocyclic $\left.\mathrm{NCHCH}_{2}\right), 34.2,31.7,26.2\left(\mathrm{t}, J_{\mathrm{CD}}=19.3 \mathrm{~Hz}, \mathrm{CHD}_{\mathrm{Et}}\right), 25.2,22.1,21.6\left(\mathrm{CH}_{2}\right), 29.4,29.3$ $\left(\mathrm{CH}^{i-\mathrm{Pr}}\right), 25.6,25.0,23.4,22.6\left(\mathrm{CH}_{3}{ }^{i-\mathrm{Pr}}\right), 10.7\left(\mathrm{CH}_{3}{ }^{\mathrm{Et}}\right)$. Assignments for the aliphatic region of the ${ }^{1} \mathrm{H}$ and ${ }^{13} \mathrm{C}$ NMR spectrum were made based on ${ }^{1} \mathrm{H}-{ }^{1} \mathrm{H}$ and ${ }^{1} \mathrm{H}-{ }^{13} \mathrm{C}$ coupling correlations of the $\mathrm{N}-\mathrm{CH}$ and $\mathrm{CH}_{3}{ }^{\mathrm{Et}}$ protons from COSY and HMBC spectra, in addition to DEFT-135 and DEPT- $90^{\circ}$ protocols; $\mathrm{MS}(\mathrm{FAB}) \mathrm{m} / z 326[\mathrm{M}]^{+}$.

\section{Crystal Structure Determination of Compounds 4b, 10a, 11a, and 18a}

The Bruker X8-APEX X-ray diffraction instrument with Mo radiation was used for data collection. All data frames were collected at low temperature $(T=100 \mathrm{~K})$ using an $\omega, \phi$-scan mode $\left(0.5^{\circ} \omega\right.$-scan width, hemisphere of reflections) and integrated using a Bruker SAINTPLUS software package. The intensity data were corrected for Lorentzian polarization. Absorption corrections were performed using the SADABS program. The SIR97 software package was used for direct methods of phase determination and the Bruker SHELXTL software package for structure refinement and difference Fourier maps. Atomic coordinates and isotropic and anisotropic displacement parameters of all of the non-hydrogen atoms of two compounds were refined by means of a full-matrix least-squares procedure on $F^{2}$. All H-atoms were included in the refinement in calculated positions riding on the $\mathrm{C}$ atoms. Drawings of molecules were performed using Ortep 3.

\section{Crystal and structure parameters of $4 \mathrm{~b}$}

size $0.51 \times 0.17 \times 0.12 \mathrm{~mm}^{3}$, monoclinic, space group P2(1)2(1)2(1), $a=10.5761$-(7) $\AA, b=$ 16.3822(10) $\AA, c=19.6147(12) \AA, \alpha=\gamma=\beta=90.0^{\circ}, V=3398.4(4) \AA^{3}, \rho_{\text {calcd }}=1.256 \mathrm{~g} /$ $\mathrm{cm}^{3}$, Mo radiation $(\lambda=0.71073 \AA), T=100(2) \mathrm{K}$, reflections collected $=67338$, independent reflections $=13087\left(R_{\text {int }}=0.0797\right)$, absorption coefficient $\mu=0.602 \mathrm{~mm}^{-1}, \max / \mathrm{min}$ transmission $=0.9313$ and $0.7488,387$ parameters were refined and converged at $\mathrm{R} 1=0.0381$, $\mathrm{wR} 2=0.0744$, with intensity $I>2 \sigma(I)$.

\section{Crystal and structure parameters of $10 a$}

size $0.40 \times 0.15 \times 0.10 \mathrm{~mm}^{3}$, monoclinic, space group $P 2(1) / \mathrm{c}, a=20.6299(18) \AA, b=19.1236$ (17) $\AA, c=13.9795(12) \AA, a=90.0^{\circ}, \beta=108.2590-(10)^{\circ}, \gamma=90.0^{\circ}, V=5237.5(8) \AA^{3}$, $\rho_{\text {calcd }}=1.199 \mathrm{~g} / \mathrm{cm}^{3}$, Mo radiation $(\lambda=0.71073 \AA), T=100(2) \mathrm{K}$, reflections collected $=24727$, independent reflections $=6333\left(R_{\mathrm{int}}=0.0322\right)$, absorption coefficient $\mu=0.163 \mathrm{~mm}^{-1}, \max /$ min transmission $=0.9839$ and $0.9378,576$ parameters were refined and converged at $\mathrm{R} 1=$ $0.0587, \mathrm{wR} 2=0.1462$, with intensity $I>2 \sigma(I)$.

\section{Crystal and structure parameters of $11 \mathrm{a}$}

size $0.46 \times 0.14 \times 0.06 \mathrm{~mm}^{3}$, monoclinic, space group $P 2(1) / \mathrm{c}, a=17.326(2) \AA, b=8.5078$ (10) $\AA, c=21.213(3) \AA, \alpha=\gamma=90.0^{\circ}, \beta=101.369(2)^{\circ}, \gamma=3065.6(6) \AA^{3}, \rho_{\text {calcd }}=1.212 \mathrm{~g} /$ $\mathrm{cm}^{3}$, Mo-radiation $(\lambda=0.71073 \AA), T=100(2) \mathrm{K}$, reflections collected $=17060$, independent reflections $=4376\left(R_{\mathrm{int}}=0.0358\right)$, absorption coefficient $\mu=0.406 \mathrm{~mm}^{-1}, \max / \mathrm{min}$ transmission $=0.9780$ and $0.8369,363$ parameters were refined and converged at $\mathrm{R} 1=0.0553$, $\mathrm{wR} 2=0.1451$, with intensity $I>2 \sigma(I)$. 


\section{Crystal and structure parameters of $18 \mathrm{a}$}

size $0.70 \times 0.19 \times 0.13 \mathrm{~mm}^{3}$, monoclinic, space group $P 2(1) / c, a=12.535(3) \AA, b=20.342$ (5) $\AA, c=27.860(7) \AA, \alpha=90.0^{\circ}, \beta=94.939(6)^{\circ}, \gamma=90.0^{\circ}, V=7077(3) \AA^{3}, \rho_{\text {calcd }}=1.398 \mathrm{~g} /$ $\mathrm{cm}^{3}$, Mo radiation $(\lambda=0.71073 \AA), T=100(2) \mathrm{K}$, reflections collected $=32838$, independent reflections $=10046\left(\mathrm{R}_{\text {int }}=0.0754\right)$, absorption coefficient $\mu=0.750 \mathrm{~mm}^{-1}, \max / \mathrm{min}$ transmission $=0.9088$ and $0.6219,823$ parameters were refined and converged at $\mathrm{R} 1=0.0538$, wR2 $=0.1277$, with intensity $I>2 \sigma(I)$.

\section{Acknowledgements}

We are grateful to the NIH (R01 GM 68825) and RHODIA for financial support.

\section{References}

1. Anastas, PT.; Williamson, TC. Green Chemistry: Frontiers in Benign Chemical Syntheses and Processes. Oxford University Press; New York: 1999. Wasserscheid, P.; Welton, T. Ionic Liquids in Synthesis. Wiley-VCH; Weinheim: 2003. (c) Dupont J, de Souza RR, Suarez PAZ. Chem Rev 2002;102:3667-3691. [PubMed: 12371898] (d) Wasserscheid P, Keim W. Angew Chem, Int Ed 2000;39:3773-3789.

2. Ohno, H. Electrochemical Aspects of Ionic Liquids. Wiley-Interscience; New York: 2005.

3. (a) Arduengo AJ III, Harlow RL, Kline M. J Am Chem Soc 1991;113:361-363. (b) Arduengo AJ III. Acc Chem Res 1999;32:913-921.

4. For reviews on NHCs, see: (a)NolanSPN-Heterocyclic Carbenes in SynthesisWiley-VCHNew York2006(b)GloriusFN-Heterocyclic Carbenes in Transition Metal Catalysis (Topics in Organometallic Chemistry)Springer-VerlagNew York2006 (c) Hahn FE. Angew Chem, Int Ed 2006;45:1348-1352. (d) Kuhn N, Al-Sheikh A. Coord Chem Rev 2005;249:829-857. (e) Peris E, Crabtree RH. Coord Chem Rev 2004;248:2239-2246. (f) Crudden CM, Allen DP. Coord Chem Rev 2004;248:2247-2273. (g) César V, Bellemin-Laponnaz S, Gade LH. Chem Soc Rev 2004;33:619636. [PubMed: 15592627] (h) Scott NM, Nolan SP. Eur J Inorg Chem 2005:1815-1828. (i) Perry MC, Burgess K. Tetrahedron: Asymmetry 2003;14:951-961. (j) Herrmann WA. Angew Chem, Int Ed 2002;41:1290-1309. (k) Bourissou D, Guerret O, Gabbaï FP, Bertrand G. Chem Rev 2000;100:39_ 91. [PubMed: 11749234]

5. See, for examples: (a) Grundemann S, Albrecht M, Kovacevic A, Faller JW, Crabtree RH. J Chem Soc, Dalton Trans 2002:2163-2167. (b) McGuinness DS, Cavell KJ, Yates BF, Skelton BW, White AH. J Am Chem Soc 2001;123:8317-8328. [PubMed: 11516281] (c) Ho VM, Watson LA, Huffman JC, Caulton KG. New J Chem 2003;27:1446-1450. (d) Clement ND, Cavell KJ, Jones C, Elsevier CJ. Angew Chem, Int Ed 2004;43:1277-1279. (e) Duin MA, Clement ND, Cavell KJ, Elsevier CJ. Chem Commun 2003:400-401. (f) Cavell KJ, McGuinness DS. Coord Chem Rev 2004;248:671-681.

6. (a) Kremzow D, Seidel G, Lehmann CW, Fürstner A. Chem Eur J 2005;11:1833-1853. (b) Schütz J, Herdtweck E, Herrmann WA. Organometallics 2004;23:6084-6086. (c) Fürstner A, Seidel G, Kremzow D, Lehmann CW. Organometallics 2003;22:907-909. (d) Fraser PJ, Roper WR, Stone FGA. J Chem Soc, Dalton Trans 1974:102-105.

7. Fürstner A, Alcarazo M, César V, Lehmann CW. Chem Commun 2006:2176-2178.

8. (a) Pandit UK, Bieraugel H. Chem Commun 1979:117-119. (b) Schipper E, Chinery E. J Org Chem 1961;26:4480-4487. (c) Hahn FE, Paas M, Le Van D, Fröhlich R. Chem Eur J 2005;11:5080-5085.

9. Arduengo AJ III, Krafczyk R, Schmutzler R. Tetrahedron 1999;55:14523-14534.

10. See for examples: (a) Paczal A, Benyei AC, Kotschy A. J Org Chem 2006;71:5969-5979. [PubMed: 16872179] (b) Jazzar R, Liang H, Donnadieu B, Bertrand G. J Organomet Chem 2006;691:32013205. (c) van Veldhuizen JJ, Campbell JE, Guidici RE, Hoveyda AH. J Am Chem Soc 2005;127:6877-6882. [PubMed: 15869311] (d) Waltman AW, Grubbs RH. Organometallics 2004;23:3105-3107. (e) Rivas FM, Riaz U, Giessert AJ, Smulik A, Diver ST. Org Lett 2001;3:26732676. [PubMed: 11506606] (f) Hahn FE, Paas M, Le Van D, Lügger T. Angew Chem, Int Ed 2003;42:5243-5246. (g) Hahn FE, Paas M, Le Van D, Frölich R. Chem Eur J 2005;11:5080-5085. (h) Xu G, Gilbertson SR. Org Lett 2005;7:4605-4608. [PubMed: 16209490] (i) Clavier H, Coutable L, Toupet L, Guillemin JC, Mauduit M. J Organomet Chem 2005;690:5237-5254. (j) Bappert E, 
Helmchen G. Synlett 2004:1789-1793. (k) Yoshida H, Sugiura S, Kunai A. Org Lett 2002;4:27672769. [PubMed: 12153230]

11. O’Hagan D. Nat Prod Rep 2000;17:435-446. [PubMed: 11072891]

12. For reviews, see: (a) Matsunaga S. J Synth Org Chem Japan 2006;64:778-779. (b) Hultzsch KC. Adv Synth Catal 2005;347:367-391. (c) Alonso F, Beletskaya IP, Yus M. Chem Rev 2004;104:30793159. [PubMed: 15186189] (d) Hong S, Marks TJ. Acc Chem Res 2004;37:673-686. [PubMed: 15379583] (e) Roesky PW, Muller TE. Angew Chem, Int Ed 2003;42:2708-2710. (f) Pohlki F, Doye S. Chem Soc Rev 2003;32:104-114. [PubMed: 12683107] (g) Muller T, Beller M. Chem Rev 1998;98:675-703. [PubMed: 11848912]

13. (a) Rosenfeld DC, Shekhar S, Takemiya A, Utsunomiya M, Hartwig JF. Org Lett 2006;8:4179-4182. [PubMed: 16956181] (b) Motokura K, Nakaagiri N, Mori K, Mizugaki T, Ebitani K, Jitsukawa K, Kaneda K. Org Lett 2006;8:4617-4620. [PubMed: 16986964] (c) Lapis AAM, DaSilveira Neto BA, Scholten JD, Nachtigall FA, Eberlin MN, Dupont J. Tetrahedron Lett 2006;47:6775-6779. (d) Li ZG, Zhang JL, Brouwer C, Yang CG, Reich NW, He C. Org Lett 2006;8:4175-4178. [PubMed: 16956180] (e) Anderson LL, Arnold J, Bergman RG. J Am Chem Soc 2005;127:14542-14543. [PubMed: 16231885] (f) Schlummer B, Hartwig JF. Org Lett 2002;4:1471-1474. [PubMed: 11975606]

14. Jazzar R, Dewhurst RD, Bourg JB, Donnadieu B, Canac Y, Bertrand G. Angew Chem, Int Ed. in press

15. (a) Lavallo V, Canac Y, Präsang C, Donnadieu B, Bertrand G. Angew Chem, Int Ed 2005;44:57055709. (b) Lavallo V, Canac Y, DeHope A, Donnadieu B, Bertrand G. Angew Chem, Int Ed 2005;44:7236-7239. (c) Lavallo V, Canac Y, Donnadieu B, Schoeller WW, Bertrand G. Angew Chem, Int Ed 2006;45:3488-3491.

16. (a) Chrzanowska M, Rozwadowska MD. Chem Rev 2004;104:3441-3370. (b) Bohe L, Kammoun M. Tetrahedron Lett 2002;43:803-805. (c) Bulman Page PC, Buckley BR, Appleby LF, Alsters PA. Synthesis 2005:3405-3411.

17. A few stable acyclic (aryl)(amino)carbenes have been reported: (a) Sole H, Gornitzka H, Schoeller WW, Bourissou D, Bertrand G. Science 2001;292:1901-1903. [PubMed: 11397943] (b) Cattoën X, Gornitzka H, Bourissou D, Bertrand G. J Am Chem Soc 2004;126:1342-1343. [PubMed: 14759183]

18. Ulrich H, Tilley JN, Sayigh AAR. J Org Chem 1964;29:2401-2404.

19. Spino C, Godbout C, Beaulieu C, Harter M, Mwene-Mbeja TM, Boisvert L. J Am Chem Soc 2004;126:13312-13319. [PubMed: 15479086]

20. Chen CL, Liu YH, Peng SM, Liu STJ. Organomet Chem 2004;689:1806-1815.

21. Bambirra S, van Leusen D, Meetsma A, Hessen B, Teuben JH. Chem Commun 2003:522-523.

22. Berreau LM, Chen J, Woo LK. Inorg Chem 2005;44:7304-7306. [PubMed: 16212351]

23. Fleming L, Woolias MJ. Chem Soc, Perkin Trans 1 1979;3:829-837. 


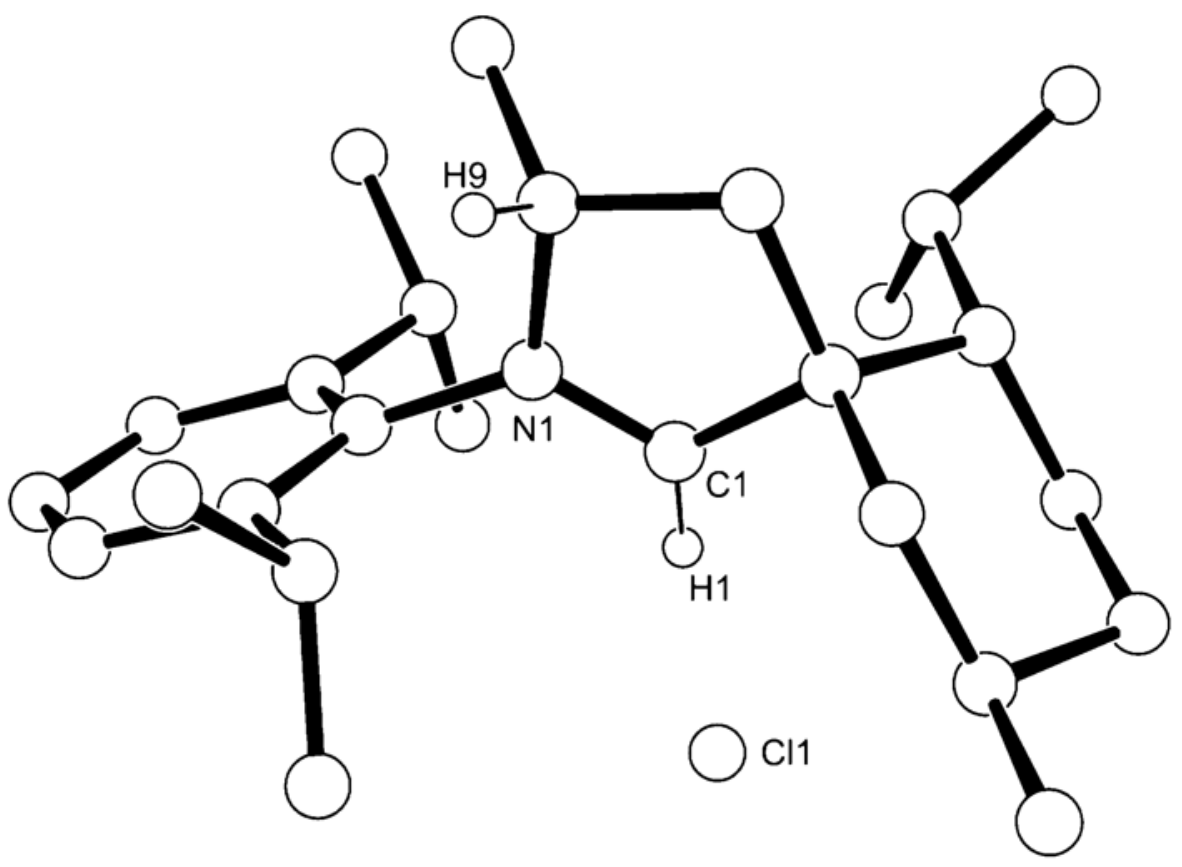

FIGURE 1.

Molecular structure of the major isomer of the cyclic aldiminium salt $\mathbf{4 b}$ in the solid state. 


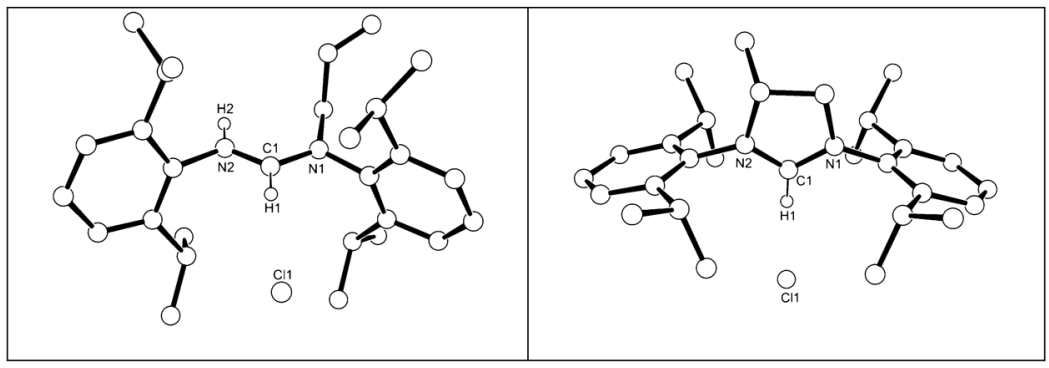

FIGURE 2.

Molecular structures of alkenyl formamidinium salt 10a (left) and imidazolinium salt 11a (right) in the solid state. 

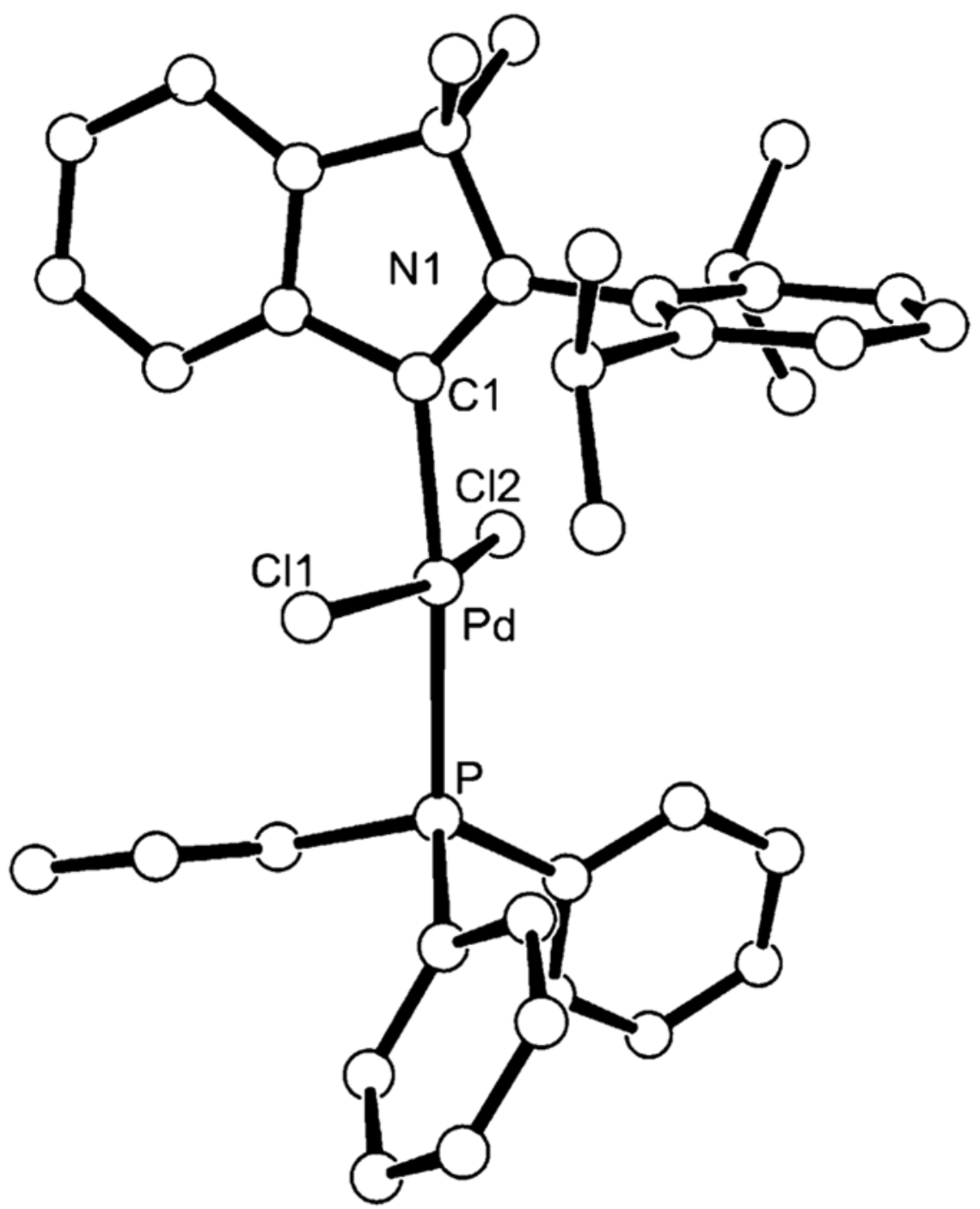

FIGURE 3.

Molecular structure of the palladium complex 18a (H atoms are omitted). Selected bond distances ( $\AA$ ) and angles (deg): N1- C1 1.307(7), C1-Pd 1.997(6), Pd-P 2.3514(16), Pd-Cl1 2.3088(15), Pd-Cl2 2.2957(15); C $\mathrm{ar}_{-}-\mathrm{C} 1-\mathrm{N} 1$ 107.8(5), C1-Pd-Cl1 88.41(15), C1-Pd-Cl2 87.18(15), C1-Pd-P 173.87(16). 

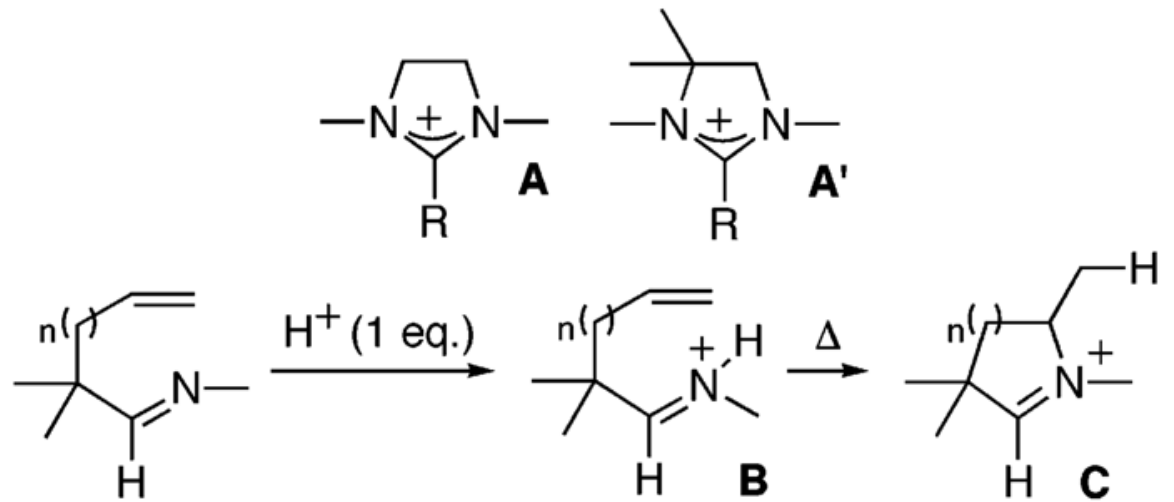

SCHEME 1. 


$$
\begin{aligned}
& \text { 2a-4a: } \mathrm{R}=\mathrm{Me} ; 2 \mathbf{b}-4 \mathbf{b}: \mathrm{R}=\mathrm{H} \\
& \text { Dipp: } 2,6-\mathrm{di} P \mathrm{PC}_{6} \mathrm{H}_{3}
\end{aligned}
$$

\section{SCHEME 2.}




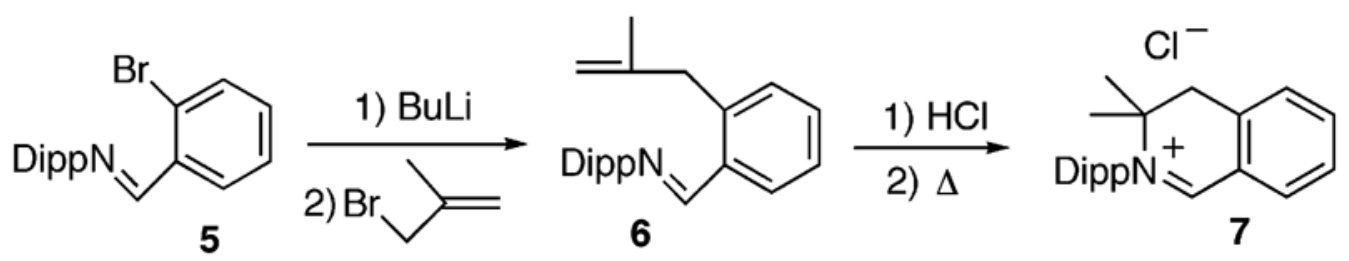

SCHEME 3. 

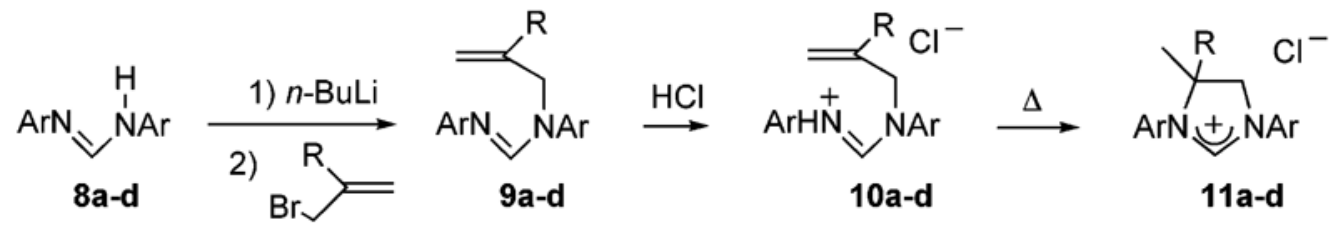

8a-11a: $A r=\operatorname{Dipp}, \mathrm{R}=\mathrm{H}$; 8b-11b: $\mathrm{Ar}=\mathrm{Dipp}, \mathrm{R}=\mathrm{Me}$; 8c-11c: $\mathrm{Ar}=\mathrm{Mes}, \mathrm{R}=\mathrm{Me}$; 8d-11d: $\mathrm{Ar}=$ 2,6-difluorophenyl, $\mathrm{R}=\mathrm{Me}$

SCHEME 4. 


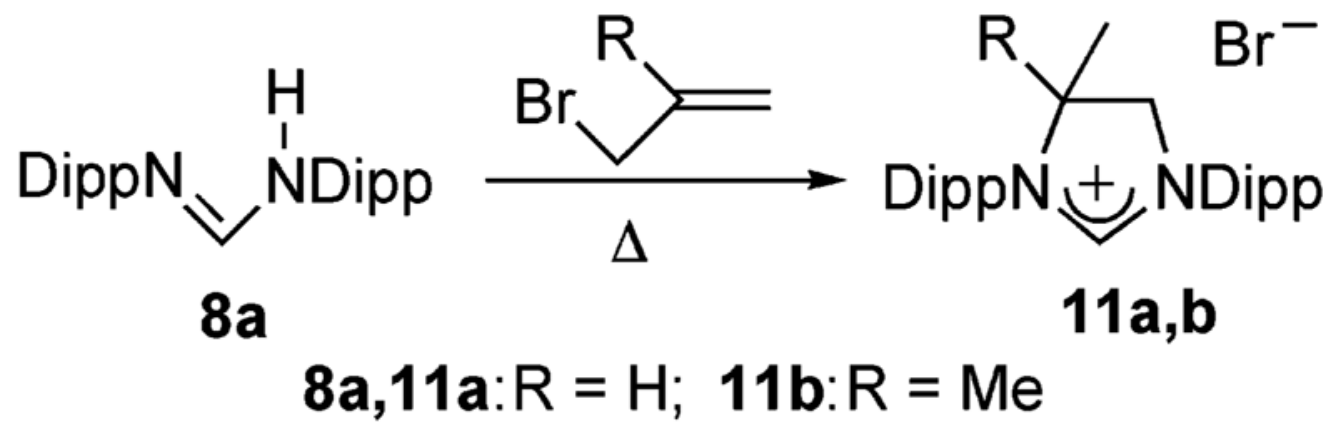

SCHEME 5. 

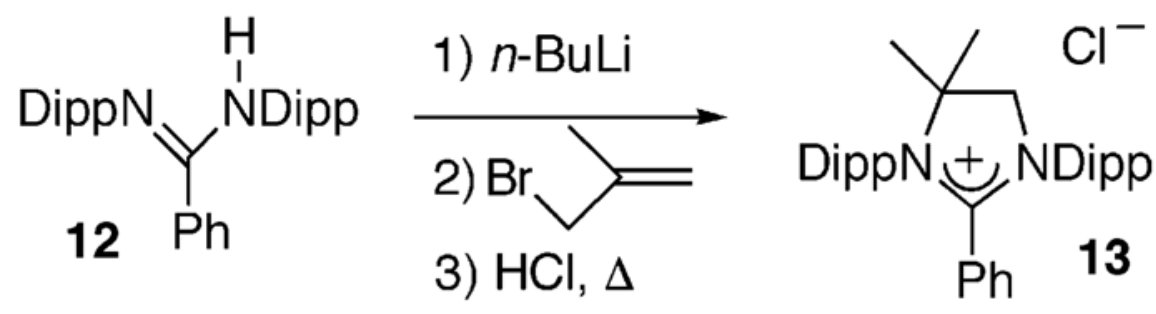

SCHEME 6. 


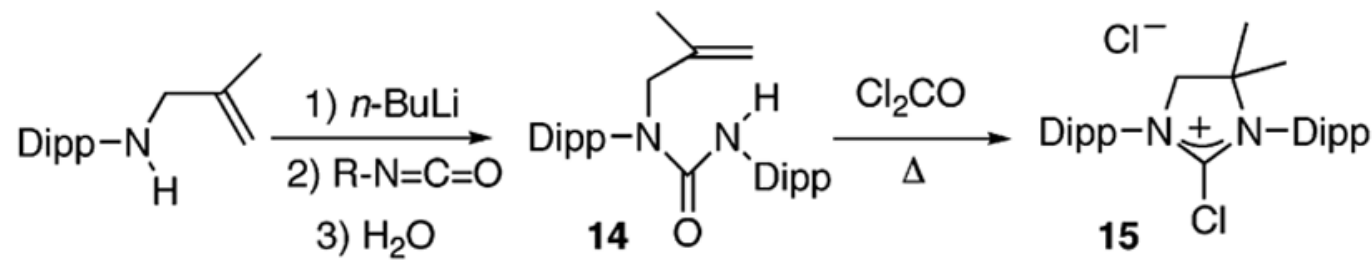

SCHEME 7. 


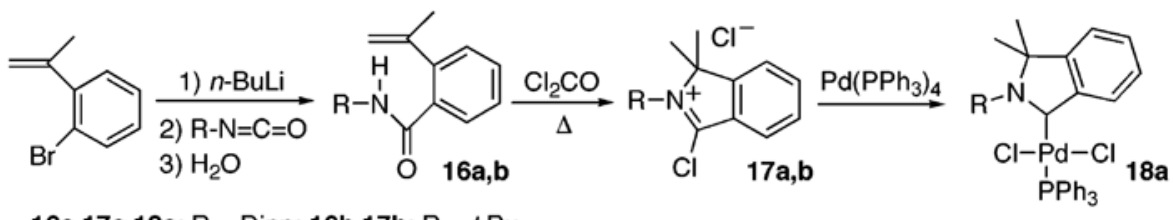
16a,17a,18a: $R=\operatorname{Dipp} ;$ 16b,17b: $R=t-B u$

SCHEME 8. 
<smiles>[2H]CC1C[N+]([O-])([O-])C[N+]1([O-])[O-]</smiles>

SCHEME 9. 